\title{
A new model suite to determine the influence of cosmic rays on (exo)planetary atmospheric biosignatures
}

\section{Validation based on modern Earth}

\author{
Konstantin Herbst ${ }^{1}$, John Lee Grenfell ${ }^{2}$, Miriam Sinnhuber ${ }^{3}$, Heike Rauer ${ }^{2}$, Bernd Heber ${ }^{1}$, Saša Banjac ${ }^{1}$, \\ Markus Scheucher ${ }^{4}$, Vanessa Schmidt ${ }^{3}$, Stefanie Gebauer ${ }^{2}$, Ralph Lehmann ${ }^{5}$, and Franz Schreier ${ }^{6}$ \\ ${ }^{1}$ Institut für Experimentelle and Angewandte Physik, Christian-Albrechts-Universität zu Kiel (CAU), 24118 Kiel, Germany \\ e-mail: herbst@physik.uni-kiel.de \\ 2 Institut für Planetenforschung (PF), Deutsches Zentrum für Luft- und Raumfahrt (DLR), Rutherfordstr. 2, \\ 12489 Berlin, Germany \\ ${ }^{3}$ Institut für Meteorologie und Klimaforschung, Karlsruher Institut für Technologie (KIT), Hermann-von Helmholtz Platz 1, \\ 76344 Eggenstein-Leopoldshafen, Germany \\ ${ }^{4}$ Zentrum für Astronomie und Astrophysik, Technische Universität Berlin (TUB), Hardenbergstrasse 36, 10623 Berlin, Germany \\ ${ }^{5}$ Alfred Wegener Institut, Helmholtz Zentrum für Polar- und Meeresforschung, Telegrafenberg A45, 14473 Potsdam, Germany \\ ${ }^{6}$ Institut für Methodik der Fernerkundung, Deutsches Zentrum für Luft- und Raumfahrt (DLR), 82234 Oberpfaffenhofen, \\ Germany
}

Received 14 May 2019 / Accepted 13 September 2019

\begin{abstract}
Context. The first opportunity to detect indications for life outside of the Solar System may be provided already within the next decade with upcoming missions such as the James Webb Space Telescope (JWST), the European Extremely Large Telescope (E-ELT) and the Atmospheric Remote-sensing Infrared Exoplanet Large-survey (ARIEL) mission, searching for atmospheric biosignatures on planets in the habitable zone of cool K- and M-stars. Nevertheless, their harsh stellar radiation and particle environment could lead to photochemical loss of atmospheric biosignatures.

Aims. We aim to study the influence of cosmic rays on exoplanetary atmospheric biosignatures and the radiation environment considering feedbacks between energetic particle precipitation, climate, atmospheric ionization, neutral and ion chemistry, and secondary particle generation.

Methods. We describe newly combined state-of-the-art modeling tools to study the impact of the radiation and particle environment, in particular of cosmic rays, on atmospheric particle interaction, atmospheric chemistry, and the climate-chemistry coupling in a selfconsistent model suite. To this end, models like the Atmospheric Radiation Interaction Simulator (AtRIS), the Exoplanetary Terrestrial Ion Chemistry model (ExoTIC), and the updated coupled climate-chemistry model are combined.

Results. In addition to comparing our results to Earth-bound measurements, we investigate the ozone production and -loss cycles as well as the atmospheric radiation dose profiles during quiescent solar periods and during the strong solar energetic particle event of February 23, 1956. Further, the scenario-dependent terrestrial transit spectra, as seen by the NIR-Spec infrared spectrometer onboard the JWST, are modeled. Amongst others, we find that the comparatively weak solar event drastically increases the spectral signal of $\mathrm{HNO}_{3}$, while significantly suppressing the spectral feature of ozone. Because of the slow recovery after such events, the latter indicates that ozone might not be a good biomarker for planets orbiting stars with high flaring rates.
\end{abstract}

Key words. astrobiology - planets and satellites: terrestrial planets - planets and satellites: atmospheres

\section{Introduction}

Cool stars such as $\mathrm{K}$ - and M-dwarfs are prime targets in the search for habitable Earth-like planets due to high occurrence rates in the solar neighborhood, small radii (leading to favorable planet-to-star contrast ratios), and rather close-in habitable zones as well as short intervals between transit events (see, e.g., Gillon et al. 2017; Dittmann et al. 2017, and references therein). Although recent results suggest that rocky planets in the habitable zone (HZ) of these host-stars could retain liquid water on their surfaces (Noack et al. 2017), their presence within the HZ does not automatically imply that they are habitable (see, e.g., Lammer et al. 2009). The close-in HZ and a possibly weakened planetary magnetic field due to potential tidal-locking (see, e.g., Scalo et al. 2007) suggest that these worlds could be subject to a large input from stellar UV photons and energetic particles modulated by their stellar wind. There are essentially two types of energetic particles: galactic cosmic rays (GCRs) from, for example, supernova remnants (e.g., Büsching et al. 2005, and references therein) and stellar cosmic rays (SCRs), which arise from stellar activity (see, e.g., Buccino et al. 2007; France et al. 2013, and references therein). The region around a star dominated by its stellar wind and stellar magnetic field, also known as astrosphere, is filled by plasma of stellar origin (see e.g., Wood et al. 2005), which protects the (exo)planet against the isotropically distributed GCRs. The extension of the astrosphere, and thus the amount of protection, is determined by the stellar wind. The (exo)planetary magnetosphere, the magnetic environment of the planet, protects against stellar energetic particles (SEPs) and the stellar wind. Factors such as the presence 
of stellar coronal mass ejections (Khodachenko et al. 2007) can lead to the erosion of unprotected planetary atmospheres. Furthermore, extreme SEP events may cause drastic radiation dose increases and thus may drastically impact life (Vidotto et al. 2013; do Nascimento et al. 2016).

Moreover, the different stellar instellation could lead to planetary surface conditions that are very different from those on Earth in terms of cosmic ray (CR) flux, radiation, and climate. For these reasons, studying the influence of stellar radiation and particle environment on planetary conditions in a physically consistent manner requires a suite of state-of-the-art modeling tools together with expertise from numerous interdisciplinary fields such as space and atmospheric physics and biogeochemical-climate modeling. Due to the complexity of the problem, limiting initial model studies to Earth-like exoplanets is quite common in the literature. In reality however, the potential range of for example atmospheres, biospheres, and interiors is ill-constrained. Furthermore, due to their relatively close-in orbits, Earth-like exoplanets around K- and M-stars could be tidally-locked to their parent star. Consequently, the presence of sufficient atmospheric (see e.g., Joshi et al. 1997) or oceanic (see, e.g., Hu \& Yang 2014) mass for meridional transport of energy from the day to the night side may be seen as a precondition for habitability. Numerous numerical 3D studies for rocky exoplanets in the $\mathrm{HZ}$ of $\mathrm{K}$ - and M-stars have recently been published (see, e.g., Leconte et al. 2013; Shields et al. 2013; Yang et al. 2013; Godolt et al. 2015; Kopparapu et al. 2016). Such studies are vital because potentially rocky exoplanets orbiting in the HZ of cool stars are already starting to be detected by combining the transit method (determining radius) with the radial velocity method (determining mass), and more are expected to be found by the upcoming space missions. Therefore, theoretical studies are required now to understand the expected range of atmospheres, interiors, climates, and spectral signatures in the context of next-generation missions such as the James Webb Space Telescope (JWST, Kaltenegger \& Traub 2009; Belu et al. 2011; Rauer et al. 2011), the European Extremely Large Telescope (E-ELT, e.g., Snellen et al. 2013), and farther-reaching missions such as the Habitable Exoplanet Observatory (HabEX) and the Large UV/Optical/IR Surveyor (LUVOIR). A summary of future exoplanet missions in an exoplanetary context can be found in Fujii et al. (2018), for example. Recent publications by Schwieterman et al. (2018) and Grenfell et al. (2018) provide comprehensive reviews of current biosignature science and photochemical gas-phase biosignature responses, respectively. Shields et al. (2016) recently reviewed the potential habitability of planets in the $\mathrm{HZ}$ of M-stars.

In summary, in order to study planetary habitability, detailed knowledge of (i) the astrospheric particle- and radiation environment, (ii) the atmospheric chemistry, and (iii) the climatechemistry coupling is mandatory. Although cool stars are favored targets for investigating rocky exoplanets, the effect of strong stellar energetic particles on potential atmospheric biosignatures is not well known.

Here we present our approach to building up a self-consistent simulation chain, coupling state-of-the-art magnetospheric and atmospheric propagation and interaction models with atmospheric chemistry and climate models in our study involving the Christian-Albrechts-Universtität (CAU), the Deutsches Zentrum für Luft- und Raumfahrt (DLR), and the Karlsruher Institute of Technology (KIT) which is supported by the German Research Foundation (DFG).

The present study focuses on the validation of our model chain based on modern Earth as an "exoplanet" in the HZ of a
G2V star during quiescent as well as constant flaring conditions. Future studies will focus on exoplanetary scenarios such as the potentially Earth-like exoplanet Proxima Centauri b.

\section{Scientific background}

\subsection{Particle propagation and transport in magnetospheres and atmospheres}

The structure of an astrosphere strongly depends on the properties of both the hot and fully ionized stellar wind as well as the surrounding interstellar medium (ISM). Differences in the properties of the stellar wind and the local ISM may thereby lead to a wide range of shapes for observable astrospheres (see, e.g., Kobulnicky et al. 2016). Simulations of astrospheres around hot stars most often make use of 1D or 2D (magneto-)hydrodynamical approaches (see, e.g., van Marle et al. 2014; Cox et al. 2016). However, in order to estimate the radiation and $\mathrm{CR}$ particle field of G-, K-, and M-stars, 3D astrospheric modeling becomes inevitable. The full-3D MHD code Cronos (see, e.g, Kissmann et al. 2008; Wiengarten et al. 2013) as well as the 3D kinetic MHD model by Izmodenov \& Alexashov (2015) have been successfully used by for example Scherer et al. (2015) and Katushkina et al. (2018) to model the astrospheres of hot O-type stars, respectively.

Moreover, the transport of GCRs through an astrosphere depends on its volume, structure, and turbulent state, which are governed by for example the stellar type, rotation rate, stellar wind dynamics, stellar activity, and magnetic field as inner boundary conditions and the local interstellar spectrum (LIS) as an outer boundary condition. In the case of the Sun, the latter however was only recently derived from measurements by the Voyager 1 spacecraft (see, e.g., Stone et al. 2013; Webber \& McDonald 2013), and several LIS models exist in the literature (an overview of the models is given by Herbst et al. 2017). By treating the astrosphere as a heliospheric analog, transport and modulation of GCRs can be investigated by applying Parker's transport equation (see Parker 1968).

An often-used approximation is the so-called Force-Field approximation (FFA) which can be applied provided the planet is located well inside the astrosphere or heliosphere (Caballero-Lopez \& Moraal 2004; Moraal 2013). This firstorder approximation of the GCR flux at a distance of $1 \mathrm{AU}$ is given by

$$
J(E, \phi)=J_{\mathrm{LIS}}(E+\phi) \cdot \frac{E \cdot\left(E+2 E_{\mathrm{r}}\right)}{(E+\Phi) \cdot\left(E+\Phi+2 E_{\mathrm{r}}\right)},
$$

where $J_{\text {LIS }}$ represents the unmodulated differential GCR flux outside the heliosphere and $E_{\mathrm{r}}$ denotes the rest energy of the primary particle (Moraal 2013). The stellar modulation function is given by $\Phi=(\mathrm{Ze} / A) \phi$ (in units of $\mathrm{GeV}$ ), with $Z$ being the charge and $A$ the mass number of the particle, while $\phi$ represents the socalled modulation parameter, a measure of the stellar activity (in units of GV). High stellar activity, for example, corresponds to strong modulation, that is, high $\phi$ values and therefore low GCR fluxes, and vice versa. According to Caballero-Lopez \& Moraal (2004), $\phi$ can be derived via

$\phi(r) \equiv \int_{P}^{P_{b}(r, P)} \frac{\beta\left(P^{\prime}\right) \kappa_{2}\left(P^{\prime}\right)}{P^{\prime}} \mathrm{d} P^{\prime}=\int_{r}^{r_{\mathrm{b}}} \frac{v\left(r^{\prime}\right)}{3 \kappa_{1}\left(r^{\prime}\right)} \mathrm{d} r^{\prime}$,

where $r_{\mathrm{b}}$ represents the outer boundary of the astrosphere, $v$ the stellar wind velocity, and $\kappa$ the diffusion coefficient. Therefore, 
in theory, the FFA can be applied to determine the GCR flux within any astrosphere. However, this approach, among others, neglects adiabatic cooling processes and can depend on additional parameters such as the magnetic field structure, particle drifts, stellar cycle, and so on (Gieseler et al. 2017, and references therein). Therefore, the application of the FFA to other stars requires knowledge of the astrospheric turbulence (represented by $\kappa)$ as well as the stellar wind velocity. Although stellar $\kappa$ values are unknown (and thus usually replaced by corresponding heliospheric values), the stellar wind velocity can be calculated with stellar wind models (see, e.g., Preusse et al. 2005; Vidotto et al. 2011). The required input parameters, such as the stellar magnetic field strength, the effective stellar temperature, and the mass flux, are frequently challenging to obtain, but can also be derived indirectly (see, e.g., Preusse et al. 2006; Kopp et al. 2011). Such models predict weaker winds, and thus lower $\phi$-values, for quiet K-stars (with its HZ centered at approximately $0.6 \mathrm{AU}$ ) but considerably stronger winds for active M-stars (assuming a $\mathrm{HZ}$ centered at approximately $0.2 \mathrm{AU}$ ) compared to our G2V star, the Sun. Consequently, the energetic particle flux at the orbit of a planet in the $\mathrm{HZ}$ around quiet K-stars may be dominated by the GCR component, whereas the SEP component is considerably more important for planets around active $\mathrm{K}$ - and M-stars due to high stellar activity and strong stellar winds.

Strong solar activity can also lead to particles that are being accelerated up to several $\mathrm{GeV}$ spiraling along the heliospheric magnetic field lines. Planets closely connected to these field lines will encounter an enhanced solar particle flux, which may be detected at the planetary surface of, for example, Earth and Mars (see, e.g., Shea \& Smart 2000). These events are so-called ground level enhancements (GLEs) and are also expected to occur in exoplanetary atmospheres. The incoming SCR flux on exoplanets in the HZ of cooler stars, however, is currently not observable. Therefore, Segura et al. (2010) proposed an indirect means to estimate the SEP flux based on a so-called peak size distribution function (PSD, see, e.g., Belov et al. 2005) reflecting the power-law relation between peak X-ray flare intensities and peak proton fluxes. However, recently, Herbst et al. (2019a) showed that estimating the proton fluxes around K- and M-stars is not as straightforward as previously thought. Based on a new double-power-law function they, for the first time, provide best- and worst-case estimates of the stellar proton fluxes around $\mathrm{K}$ - and M-dwarfs, indicating that previous studies may have underestimated the stellar particle fluxes.

Additionally, planetary magnetospheres act as a filter for low-energy particles. Therefore, the cosmic ray (CR) flux at the top of the atmosphere (TOA) depends on the magnitude and geometry of the planetary magnetic field (Fichtner et al. 2013; Herbst et al. 2013). However, because rocky exoplanets in the HZ around M-stars may be tidally locked, weaker exoplanetary magnetic fields could be present (see, e.g., Christensen \& Aubert 2006; Grießmeier et al. 2009). In addition, Cohen et al. (2014) suggested that the magnetosphere structure of habitable planets orbiting M-dwarfs could be vastly different from that of the Earth; for example, with potentially strong Joule heating due to strong stellar winds impinging on the upper planetary atmosphere.

Nevertheless, an energetic charged particle entering the TOA will lose its energy within an atmosphere mostly due to collisions, leading to an atmospheric ionization, dissociative ionization, excitation, and dissociation of the most abundant species in the upper layers. Figure 1 shows schematically the incoming trajectory of a CR particle (solid red line) penetrating the magnetic field of the Earth. Besides ionizing the upper layers, interactions

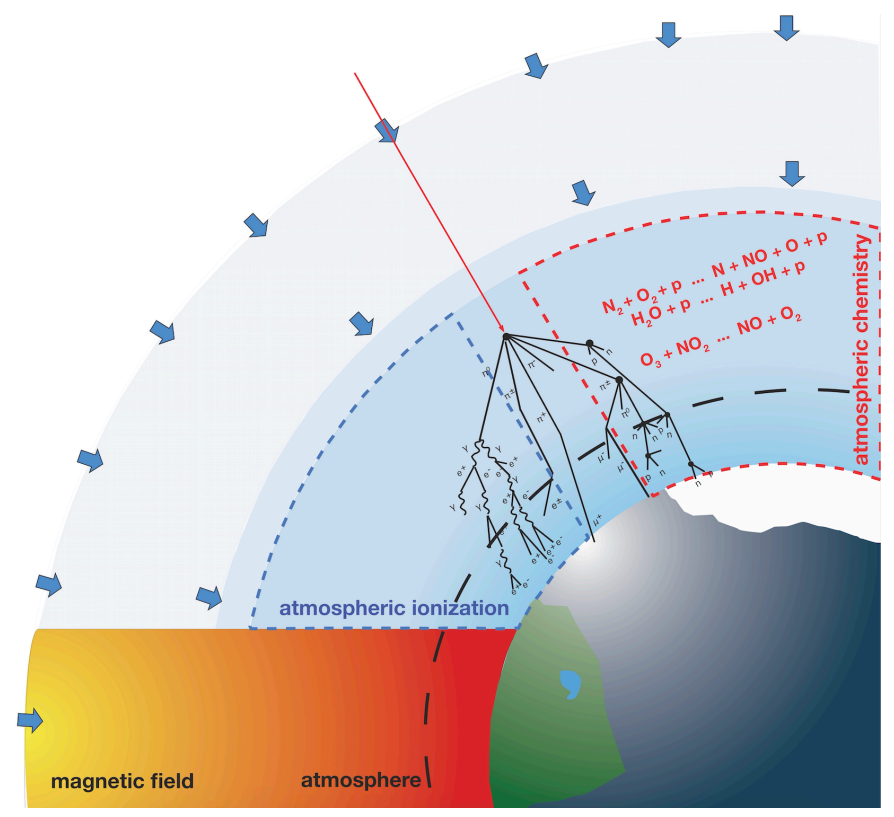

Fig. 1. Processes induced by cosmic rays affecting the atmospheric climate and ion-relevant chemistry of an Earth-like exoplanet.

with the atmospheric constituents like nitrogen, oxygen, or argon trigger the evolution of a secondary particle shower as well as associated photochemical effects (see below). The probability of an energetic CR particle colliding with atmospheric species increases with atmospheric depth. The resulting energetic secondary particles may partake in further interactions forming an atmospheric particle cascade (Dorman et al. 2004). A significant quantity describing the effect of energetic particles on life is the radiation dose (Horneck 2001; Zeitlin et al. 2011), which depends on the particle species. Furthermore, electrons created in the secondary particle showers may lead to a breakup of molecular nitrogen and oxygen followed by ion and gas-phase chemistry that produces nitrogen and hydrogen oxides. These, in turn, can catalytically remove atmospheric ozone, and therefore lead to enhanced harmful UV radiation reaching the surface. Recent results for the impact of particle precipitation on the formation of secondary particles, ionization, and radiation dose in the terrestrial atmosphere have been discussed in for example Mironova et al. (2015) and Banjac et al. (2019a).

A unified simulation code that can take into account the stellar radiation and particle environment, and model the particle interactions from the magnetosphere, through the ionosphere, and down to the surface has been rather lacking in the literature. Recently, however, Banjac et al. (2019a) developed the Geant4-based Atmospheric Radiation Interaction Simulator (AtRIS) code with which diverse planetary configurations can be realized. Initial validations on the planetary atmospheres of Venus (thick $\mathrm{CO}_{2}$-dominated atmosphere, see Herbst et al. $2019 b)$, Earth $\left(\mathrm{N}_{2}-\mathrm{O}_{2}\right.$-dominated atmosphere, see Banjac et al. 2019a), and Mars (thin $\mathrm{CO}_{2}$-dominated atmosphere, see Guo et al. 2019) have been performed.

\subsection{Cosmic-ray-induced ion chemistry}

Figure 2 schematically shows ion-related chemical processes induced by GCRs and SCRs. Energetic particles precipitating into the atmosphere collide with atmospheric constituents, leading to excitation, dissociation, and ionization of the most abundant species. In an $\mathrm{N}_{2}-\mathrm{O}_{2}$ atmosphere such as on 


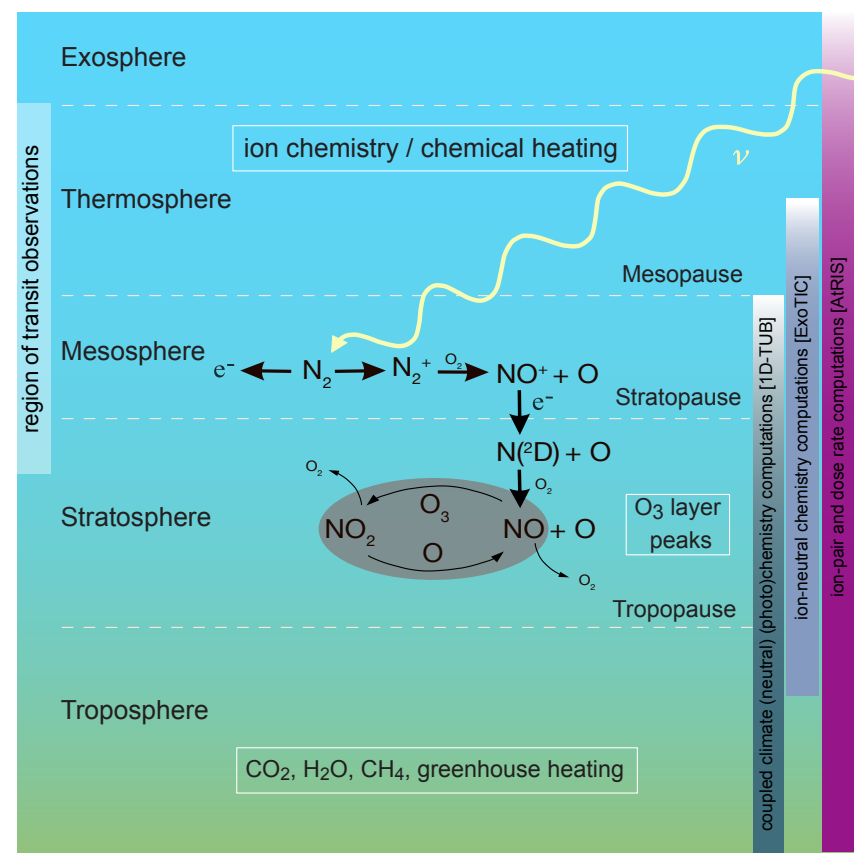

Fig. 2. Schematic showing altitude-dependent chemical interactions within an Earth-like atmosphere. The highlighted altitudes show the composition- and wavelength-dependent region typically sampled by transmission spectroscopy. In addition, we highlighted the regions at which the models of our model suite (discussed in Sect. 4) operate.

present-day Earth, this leads to the production of for example excited atomic states such as $\mathrm{N}\left({ }^{2} \mathrm{D}\right)$ and $\mathrm{O}\left({ }^{1} \mathrm{D}\right)$, or ions such as $\mathrm{N}_{2}^{+}, \mathrm{O}_{2}^{+}$, or $\mathrm{O}^{+}$. In $\mathrm{CO}_{2}$-dominated atmospheres, ions and excited-state dissociation products of $\mathrm{CO}_{2}$ would likely be the primary products. The ionization of the atmosphere leads to chains of very fast ion-chemistry reactions, which in Earth-like atmospheres lead to the formation of neutral reactive radicals such as $\mathrm{NO}, \mathrm{H}$, and $\mathrm{OH}$. These contribute to catalytic reaction chains which overall destroy ozone such as that shown for example in Eqs. (3) and (4):

$\mathrm{NO}+\mathrm{O}_{3} \longrightarrow \mathrm{NO}_{2}+\mathrm{O}_{2}$

$\mathrm{NO}_{2}+\mathrm{O}\left({ }^{3} \mathrm{P}\right) \longrightarrow \mathrm{NO}+\mathrm{O}_{2}$.

Large ion clusters can form by incorporating molecules such as $\mathrm{HNO}_{3}, \mathrm{~N}_{2} \mathrm{O}_{2}$, or $\mathrm{HCl}$, hence changing the partitioning of nitrogen- and chlorine-containing species; this will also affect the ozone content. Since ozone is the main contributor to radiative heating of the stratosphere, this directly affects atmospheric temperatures and circulation. The atmosphere of the Earth has been reviewed, for example, by Sinnhuber et al. (2012).

Ion chemistry, however, can also produce hydrocarbon haze from methane. This is of particular importance for methane-rich atmospheres like those of Jupiter and Titan (see, e.g., Vuitton et al. 2007; Lavvas et al. 2013; Hörst 2017; García Muñoz et al. 2018). In addition, methane-rich and $\mathrm{N}_{2}$-dominated atmospheres can be seen as natural laboratories to study chemical evolution (see, e.g., Kobayashi et al. 2017) and its importance for prebiotic chemistry (see, e.g., Rimmer \& Rugheimer 2019).

\subsection{Atmospheric biosignatures}

We provide here a short discussion on some of the atmospheric biosignatures commonly discussed in the exoplanetary literature. We note that this list is not exhaustive.
Ozone $\left(\mathrm{O}_{3}\right)$. Around $90 \%$ of Earth's $\mathrm{O}_{3}$ is found in the stratosphere; the remaining $10 \%$ lies in the troposphere. The terrestrial ozone is produced mainly through the Chapman mechanism (Chapman 1930) via $\mathrm{O}_{2}$ photolysis in the stratosphere and in the troposphere via the smog mechanism (Haagen-Smit 1952), which requires volatile organic compounds, nitrogen oxides, and UV radiation. Ozone is therefore an indirect biosignature of life (i.e., the direct biosignature is $\mathrm{O}_{2}$ ). Nevertheless, $\mathrm{O}_{3}$ is often focused upon in exoplanetary biosignature studies due to strong spectral absorption and because its atmospheric abundance remains relatively constant over a wide range of $\mathrm{O}_{2}$ abundances. Ozone sinks can be rather complex and include for example catalytic cycles involving $\mathrm{NO}_{x}$ and $\mathrm{HO}_{x}$ (Bates \& Nicolet 1950; Crutzen 1970). Photochemical modeling responses are discussed in Sect. 3.

Nitrous oxide $\left(\mathrm{N}_{2} \mathrm{O}\right)$. In the atmosphere of Earth $\mathrm{N}_{2} \mathrm{O}$ is formed almost exclusively as a by-product of the respiration of nitrifying and denitrifying bacteria as part of Earth's nitrogen cycle (Syakila \& Kroeze 2011). It has only very weak abiotic sources and is therefore a good biosignature. However, its spectral features in the atmosphere of modern Earth are rather weak (see, e.g., Des Marais et al. 2002; Vasquez et al. 2013b; Schreier et al. 2018b) and so it may be challenging to detect in exoplanetary atmospheres. In the stratosphere of Earth, it is removed mainly via photolysis and reactions with electronically excited oxygen atoms $\left(\mathrm{O}^{*}\right)$. Nitrous oxide can also be produced in the upper atmosphere via ionization of $\mathrm{N}_{2}$ by solar activity (see the summary in Sinnhuber et al. 2012), resulting in a possible "false positive" signal.

Methane $\left(\mathrm{CH}_{4}\right)$. On Earth, $\mathrm{CH}_{4}$ has significant biological sources in methanogenic bacteria, although it also features somewhat weaker (by one order of magnitude) abiotic sources, for example, from outgassing. Methane is a key greenhouse gas and is mainly removed via oxidative degradation from the atmosphere by the reactive hydroxyl $(\mathrm{OH})$ radical. An overview of the global atmospheric budget inventory of methane is provided by Wahlen (1993), for example.

Chloromethane $\left(\mathrm{CH}_{3} \mathrm{Cl}\right)$. Chloromethane has atmospheric sources associated with, for example, seaweed emissions. It is removed for example via photolysis and by the reactive $\mathrm{OH}$ radical (see Keppler et al. 2005).

\section{3. (Exo)planetary climate-chemistry modeling}

This section deals with the incoming stellar radiation which interacts radiatively and photochemically as it passes through the planetary atmosphere. Numerical models (described below) are constructed to solve the central equations for radiative transfer, convection, and photochemistry. The key model outputs are vertical profiles of temperature, radiative fluxes, water (for climateconvection) and chemical abundances (for (photo)chemistry).

\subsection{Earth-like planets in the $H Z$ of $M$-stars}

One-dimensional modeling studies, for example, by Segura et al. (2005), Rauer et al. (2011), and Rugheimer et al. (2015), applied a global mean, cloud-free, coupled chemistry-climate column model to investigate biosignature responses for planets orbiting a range of M-dwarfs. Results suggested significant chemical responses like, for example, a strong $\mathrm{CH}_{4}$ greenhouse effect. However, these models did not include the effects of potentially 
high SEP levels, which are expected to be essential for Earth-like planets orbiting M-dwarfs.

Segura et al. (2010) and most recently Tilley et al. (2019) used a proxy (based on X-ray observations, as described above) to calculate the $\mathrm{NO}_{x}$ production from SEP interaction with an Earth-like atmosphere (defined here as $\mathrm{N}_{2}-\mathrm{O}_{2}$-dominated), which they applied interactively in their global-mean coupled climate-chemistry column model and studied the effect on $\mathrm{O}_{3}$ for Earth-like planets in the HZ of M-dwarfs. Their results suggest that $\mathrm{O}_{3}$ may not survive strong SEP events during flaring conditions due to catalytic losses by $\mathrm{NO}_{x}$.

Grenfell et al. (2012) also investigated the effects of SEPs, confirming that atmospheric $\mathrm{O}_{3}$ may not survive extreme flaring cases for Earth-like planets orbiting active M-dwarfs. Furthermore, Tabataba-Vakili et al. (2016) studied such effects with a similar model version but updated with an additional energetic particle-induced $\mathrm{HO}_{x}$ source. In their work, a parameterization was introduced into the chemistry module simulating the breakup of molecular oxygen by SCRs which can then trigger ion cluster chemistry involving $\mathrm{H}_{2} \mathrm{O}$ to ultimately produce $\mathrm{HO}_{x}$, a sink for biosignatures such as $\mathrm{O}_{3}$. Rauer et al. (2011) investigated theoretical spectral signals of Earth-like planets in the $\mathrm{HZ}$ of M-dwarfs (without considering energetic particles) for a range of stellar spectral classes and Earth-like planets with up to $3 \mathrm{~g}$, corresponding to about ten Earth masses. Results revealed important couplings between chemistry and climate which arose for example from middle atmosphere heating from enhanced planetary abundances of the greenhouse gas $\mathrm{CH}_{4}$, especially for scenarios considering the cooler M-dwarfs that led to weakened biosignature spectral bands of $\mathrm{O}_{3}$, for example. As a follow-up, Grenfell et al. (2013a) investigated the photochemical responses for the same scenarios as Rauer et al. (2011). Results suggested that the mechanism for $\mathrm{O}_{3}$ production switched from the Chapman mechanism to the smog mechanism for the cooler stars (which emit less (E)UV and therefore slow the Chapman mechanism since this proceeds via the photolysis of the strong $\mathrm{O}_{2}$ molecule). In addition, Grenfell et al. (2013b) showed that varying the (uncertain) UV output of cool M-dwarfs could impact the photochemistry and have a potentially large effect on the $\mathrm{O}_{3}$ spectral features. They also confirmed that $\mathrm{N}_{2} \mathrm{O}$ spectral features could be enhanced for planets orbiting M-dwarfs.

Presently, both 1D and 3D models are applied in the literature to investigate atmospheres of tidally locked planets in the $\mathrm{HZ}$ of M-dwarfs. The two approaches are complementary since on the one hand 1D studies are useful to understand the wide and mostly unconstrained parameter range for exoplanet atmospheres, whereas 3D effects will play a central role because, for example, tidally locked planets could feature strong 3D transport cells from the day- to the nightside (e.g., Joshi 2003). More recently, Joshi \& Haberle (2012), Leconte et al. (2013), Shields et al. (2013), Yang et al. (2013), and Godolt et al. (2015), for example, applied 3D models to study rocky exoplanets in the HZ of K- and M-dwarfs.

Moreover, the photochemical effects of GCRs upon biosignatures were studied by Grenfell et al. (2007), for example. The results of these latter authors, however, suggest that the effects were quite modest for the scenarios investigated. For their study, they updated the standard column model chemistry module with a theoretical air shower approach to include the effects of energetic particles including related $\mathrm{NO}_{x}$ sources. Their results suggest that atmospheric biosignatures like $\mathrm{O}_{3}$ and $\mathrm{N}_{2} \mathrm{O}$ are mostly robust to GCRs; for example, column $\mathrm{O}_{3}$ decreased by only $\sim 20 \%$.

\subsection{Chemistry-climate effects in the atmosphere of modern Earth}

At Earth, the impact of SCRs on the chemical composition of the atmosphere above $20 \mathrm{~km}$, and particularly on nitrogen species and ozone, is well documented from satellite observations spanning back to the 1970s (see, e.g., Crutzen et al. 1975; Swider \& Keneshea 1973; Solomon \& Crutzen 1981; Solomon et al. 1983; Jackman et al. 1980, 1990, 2000, 2005b,a; Rohen et al. 2005; Funke et al. 2011). Ozone is the key species with respect to radiative heating in the terrestrial stratosphere. It is involved in important feedback: for example, coupling ozone heating with stratospheric temperatures and dynamical phenomena such as the Brewer-Dobson meridional circulation with its downward transport in the winter-spring polar 3D vortex on Earth can have a significant effect on the resulting $\mathrm{O}_{3}$ loss (Sinnhuber et al. 2003; Jackman et al. 2009). Model studies using chemistry-transport models (CTMs) or chemistry-climate models (CCMs) including parameterizations for $\mathrm{NO}_{x}$ and $\mathrm{HO}_{x}$ production due to atmospheric ionization have been compared with simultaneous satellite observations from satellite instruments such as MIPAS on ENVISAT in a number of dedicated model-measurement intercomparison studies (e.g., Funke et al. 2011, 2017). Key issues include the onset, distribution, and recovery time of the CR induced photochemical ozone loss. Generally, good agreement has been achieved in most studies between the observed and modeled $\mathrm{NO}_{x}$ production and ozone loss during strong particle events (e.g., Funke et al. 2011). However, recent observations and model studies also indicate a significant impact of complex ion-chemistry reactions during larger atmospheric ionization events (Sinnhuber et al. 2012; Verronen \& Lehmann 2013). The impact of energetic particle ionization on chemical composition and dynamics (for the case of Earth) is summarized in Sinnhuber et al. (2012).

\section{Simulation models used and combined in this study}

\subsection{Particle transport and interaction model: the Atmospheric Radiation Interaction Simulator}

The Atmospheric Radiation Interaction Simulator (AtRIS) was recently developed by Banjac et al. (2019a) to address the requirements necessary to investigate ionization in exoplanetary atmospheres. With AtRIS, the user can compute the hadronic and electromagnetic interactions of energetic particles with planetary atmospheres based on the GEneration ANd Tracking of particles version 4 (Geant4) code. A highlight feature is the customized planetary specification format (PSF), which provides a simple yet extremely flexible interface for the specification of the exoplanetary soil and atmosphere. This code enables simulations of (amongst other things) (i) the atmospheric secondary particle flux, (ii) the energy loss (both in the atmosphere and on the ground), and (iii) the radiation dose on the surface of an arbitrary exoplanet over a wide variety of conditions.

\subsubsection{Short description of AtRIS}

There are ongoing efforts in the literature to improve our understanding of both the electromagnetic radiation (by, e.g., the MUSCLES and MEGA-Muscles project, see France et al. 2016; Froning et al. 2018, respectively) and the flux of stellar superand hyperevents (see, e.g., Youngblood et al. 2017; Herbst et al. 2019a) experienced by rocky exoplanets orbiting M-dwarfs and 
K-stars. In the immediate future, we will not be able to measure precise primary TOA particle spectra for exoplanets. This was a starting assumption upon which the design of AtRIS was based. Instead of simulating the effect that a specific primary particle spectrum has on the exoplanetary atmosphere, AtRIS calculates three so-called atmospheric response matrices of the form $[M]_{m \times n}$, which quantify the relation between the energy (and species) of the primary particle and the ensuing altitudedependent ionization, absorbed dose, and dose equivalent. In this context the dose equivalent $H$ reflects the radiation hazard to the human body by taking into account radiation-type dependent biological effectiveness given by $H=\sum_{i} W_{i} \cdot D_{i}$, where $W_{i}$ denotes the radiation weighting factor (see ICRP 2007) and $D_{i}$ the absorbed dose due to particles of type $i$. The $m$ rows of the matrices correspond to different altitudes (atmospheric shells), while the $n$ columns correspond to different primary energies. Each matrix element describes the average of the specific quantity per particle. A column $[n]$ of the ionization ARM describes how a primary particle of such specific kinetic energy would, on average, deposit its energy at every single altitude throughout the atmosphere. Analogously, each matrix row $[m]$ describes how different primary energies contribute to the ionization at that specific altitude.

By multiplying the matrix $M$ with a vector $[N], j \in$ $\{1, \ldots, n\}$, where $N_{j}$ describes the number of primary particles in the $j$ th energy bin, we can quickly derive ionization, absorbed dose, and dose equivalent for any given input spectrum. This approach confers the advantage of being able to calculate the quantities of interest for different spectral shapes relatively quickly. Furthermore, we can investigate the effects of a planetary magnetosphere and stellar modulation by imposing either a hard or a soft energy filter on the primary spectral vector $N$.

AtRIS has been published under a general public license and includes extensive online documentation in the form of a wiki page (Banjac 2018). This offers the unique opportunity for the exoplanetary community to make use of the above-described flexibility and unique features of AtRIS in order to achieve a better understanding of the exoplanetary atmospheric ionization and radiation environments.

\subsubsection{Validation of AtRIS}

Banjac et al. (2019a,b) and, most recently, Banjac et al. (2019c) validated AtRIS for the following Earth-bound measurements: (i) ion-electron production rate (Neher 1971), (ii) surface neutron spectrum (Gordon et al. 2004), (iii) surface muon spectra (Kremer et al. 1999; Haino et al. 2004), (iv) balloon-borne proton spectrum (Haino et al. 2004), and (v) absorbed and equivalent dose rates measured on a stratospheric-balloon (Möller 2013). Further studies for Mars and Venus have been published by Guo et al. (2019) and Herbst et al. (2019b), respectively.

\subsection{Magnetospheric transport}

Since AtRIS does not implement the geomagnetic field, we used PLANETOCOSMICS (Desorgher et al. 2006), which has previously been applied to study the effect of geomagnetic field geometry upon particle propagation to the surface of Earth (see, e.g., Fichtner et al. 2013; Herbst et al. 2013). The following magnetic fields are implemented: the international geomagnetic reference field (IGRF), various magnetic dipole fields, Mars-like crustal fields, and more advanced models taking into account the interaction with the (possibly strong) stellar wind, like the model by Tsyganenko \& Stern (1996) (TSY96), which uses the stellar wind speed and magnetic field strength as input parameters. Based on these, we simulated the location and altitude-dependent cutoff rigidity values, which are a measure of the energy a particle must have in order to reach a specific point in the planetary atmosphere. The TOA proton flux is calculated depending on the cutoff rigidity.

\subsection{Ion-neutral chemistry model}

The Exoplanetary Terrestrial Ion Chemistry model (ExoTIC) is an adapted version of the University of Bremen Ion Chemistry column model (UBIC). The latter is a state-of-the-art 1D stacked box model of the neutral and ion atmospheric composition originally developed in the group of M. Sinnhuber at the University of Bremen to investigate the impact of energetic particle precipitation on ion and neutral chemistry in the terrestrial D- and E-region (Winkler et al. 2009; Sinnhuber et al. 2012; Nieder et al. 2014). It is now maintained and further developed at the KIT. Neutral and ion photochemistry is driven by photo-absorption in the UV, visible, and near-infrared (NIR) range (120-800 nm) as well as by photo-ionization in the EUV range and by particle impact ionization. Photolysis and photoionization rates are calculated from the stellar spectrum provided, and particle impact ionization rates need to be prescribed. The primary charge due to the atmospheric ionization is distributed amongst primary ions depending on the energy range of the ionization sources as well as on the atmospheric composition. The UBIC model considers 60 neutral and 120 charged species, which interact due to neutral, neutral-ion, and ion-ion gas-phase reactions, as well as photolysis and photo-electron attachment and detachment reactions (see, e.g., Sinnhuber et al. 2012). Temperature and pressure as a function of altitude also need to be prescribed; the vertical coverage of the model depends on the availability of temperature and pressure profiles. The ExoTIC model extends the applicability of UBIC to atmospheres of terrestrial (rocky) planets other than Earth.

Within our model suite, particle impact ionization rates will be prescribed by output from the AtRIS code as a function of the activity of the star. Temperature and pressure profiles are provided by the output from the Coupled 1D Terrestrial Climate-Chemistry Model (1D-TUB, see below).

In our study, the effects of ion chemistry from the ExoTIC model are implemented into the 1D-TUB model as follows: production and loss rates due to ion chemistry from ExoTIC affecting a range of neutral species are implemented into the 1D-TUB model (see also Nieder et al. 2014). The ExoTIC rates are calculated specifically for the composition and temperaturepressure profiles output by the 1D-TUB model for a given iteration and then provided as input for that model for the next iteration (see discussion in Sect. 5). In this way, atmospheric profiles for rates of change due to ion chemistry for the following ten species relevant for $\mathrm{HO}_{x}$ and $\mathrm{NO}_{x}$ responses were implemented from ExoTIC into the 1D-TUB model: $\mathrm{NO}, \mathrm{NO}_{2}, \mathrm{NO}_{3}$, $\mathrm{HNO}_{3}, \mathrm{~N}^{4} \mathrm{~S}, \mathrm{~N}^{2} \mathrm{D}, \mathrm{H}, \mathrm{OH}, \mathrm{O}$ and $\mathrm{O}^{1} \mathrm{D}$. Future work will consider additional species.

\subsection{Coupled 1D Terrestrial Climate-Chemistry Model}

We use a 1D, steady-state, cloud-free radiative-convective module to calculate atmospheric temperatures and water content, coupled to a photochemistry module calculating the steady-state mixing ratios of 55 species with over 200 reactions. This model, dating back to Kasting et al. (1984), Pavlov et al. (2000), and Segura et al. (2003), has been subsequently developed within the 
DLR/TUB Berlin group (Grenfell et al. 2011; Rauer et al. 2011; von Paris et al. 2016; Tabataba-Vakili et al. 2016; Gebauer et al. 2017, 2018b; Scheucher et al. 2018).

Please note that we do not explicitly include ion chemistry in the 1D-TUB climate-chemistry column model. Therefore, we do not include ion reactions in our chemical network since this model was initially developed for the lower layers of the atmosphere of Earth (in particular the mesosphere and below) where ion chemistry is not typically significant. The effects of ion chemistry, however, were parameterized in our model by using input from the partner model ExoTIC (see description above).

\subsubsection{Climate module}

The climate module of 1D-TUB discretizes the planetary atmosphere into 101 layers equally spaced in $\log (\mathrm{p})$ from the surface up to $1 \mathrm{~Pa}$. This region was chosen in order to simulate characteristic radiative photochemical processes in the neutral (lower to the middle) atmosphere of Earth ranging from the troposphere and stratosphere up to the mesosphere. The radiative-convective scheme is adapted from Toon et al. (1989) for the shortwave stellar absorption and Rayleigh scattering. The Modified RRTM for Application in $\mathrm{CO}_{2}$-dominated atmospheres (MRAC, von Paris et al. 2016) using the correlated-k approach is used for thermal molecular absorption by $\mathrm{H}_{2} \mathrm{O}, \mathrm{CO}_{2}, \mathrm{CH}_{4}$, and $\mathrm{O}_{3}$. For the lower atmosphere, convective adjustment to a wet adiabatic profile is performed, and the water profile is parameterized following Manabe \& Wetherald (1967). The model calculates 1D column global-average, cloud-free conditions, although cloud effects are considered straightforwardly by adjusting the surface albedo until the mean surface temperature of the Earth $(288.15 \mathrm{~K})$ is achieved for the Earth control scenario.

\subsubsection{Chemistry module}

The photochemistry module was adapted from Segura et al. (2003) for various planetary conditions. The chemistry module of 1D-TUB calculates the atmospheric conditions on the same pressure layers from the climate module minus the surface layer, giving 100 layers in total. Gebauer et al. (2017) introduced variable $\mathrm{O}_{2}$ content for the atmosphere of early Earth, while Gebauer et al. (2018b) updated the $\mathrm{O}_{2}$ photolysis rate. For the photochemistry effects induced by the precipitating high-energy particles (GCRs, SEPs) we have two possibilities, namely (i) an air shower approach using the Gaisser-Hillas method, as discussed in Tabataba-Vakili et al. (2016) and further developed as described in Scheucher et al. (2018), and (ii) direct processing of the CR-induced ionization rate of the atmosphere as calculated by AtRIS and the CR-induced chemical production rates of atmospheric molecules calculated by ExoTIC. In both approaches, the photochemistry module then incorporates atmospheric profiles of these elaborate CR-induced changes in composition. The chemistry routine also includes biogenic and source gas emissions such that the surface abundances of modern-day Earth can be reproduced.

\subsection{Diagnostic tools}

\subsubsection{The pathway analysis program}

The pathway analysis program (PAP) identifies and quantifies chemical pathways that produce or destroy a chemical species of interest. The PAP takes species concentrations and reaction rates provided by a chemical model as input. Operating step-by-step (i.e., one species after another), PAP links reactions that form a given chemical species with reactions that destroy it. By specifying $\mathrm{O}_{3}$ as the species of interest, we can apply PAP to identify and quantify $\mathrm{O}_{3}$-producing pathways as well as catalytic cycles that destroy $\mathrm{O}_{3}$. The PAP also determines the corresponding rates, which allow us to assess the importance of individual cycles at different locations (altitudes). For instance, catalytic cycles involving nitrogen oxides, chlorine oxides, and hydrogen oxides are well known to destroy $\mathrm{O}_{3}$ from the lower to the upper stratosphere of the Earth (e.g., Jucks et al. 1996). However, PAP can also be applied to conditions that differ significantly from those on Earth, and is therefore an invaluable tool for understanding complex $\mathrm{O}_{3}$ chemical responses in exoplanetary atmospheres. The PAP was developed by Lehmann (2004) and applied by Grenfell et al. (2007) to the stratosphere of Earth, by Stock et al. (2012) to the Martian atmosphere, by Grenfell et al. (2013a) to Earth-like exoplanets in the HZ around M-stars, by Gebauer et al. (2017, 2018b) to early-Earth and early Earth analog planets, and by Gebauer et al. (2018a) to early Earth analogs around M-stars.

\subsubsection{The Generic Atmospheric Radiation Line-by-line Infrared Code}

The Generic Atmospheric Radiation Line-by-line Infrared Code (GARLIC, Schreier et al. 2014) was originally developed for remote sensing the Earth's atmosphere and, more recently, was used extensively for exoplanetary studies (e.g., Rauer et al. 2011; Scheucher et al. 2018). The core of the subroutines of GARLIC constitutes the basis of forward models used to implement inversion codes to retrieve atmospheric state parameters from limb and nadir sounding instruments. GARLIC has been verified in several intercomparison studies (e.g., Schreier et al. 2018a) and validated against spectra observed on Earth (Schreier et al. 2018b) and from Venus (Hedelt et al. 2013; Vasquez et al. 2013a). GARLIC uses molecular line data from the HITRAN or GEISA database (Gordon et al. 2017; Jacquinet-Husson et al. 2016) with optional corrections for continuum and collision-induced absorption and calculates theoretical atmospheric spectra (emission, transmission, effective height) based on the converged output (climate and abundance profiles) from 1D-TUB.

\section{Building up a self-consistent simulation chain}

Figure 3 sketches the interplay between the modules of our coupled model suite and the goals that we wish to achieve. Close interaction between the three groups involved (KIT, TUB, and $\mathrm{CAU}$ ) is mandatory. Additional interfaces to address atmospheric climate and composition as well as the assessment and parameterization of ion-chemical impacts between models have been developed. The following steps have to be performed: (1) the measured stellar UV-fluxes, as well as the estimates of the stellar wind velocities, stellar modulation, and PSDs (CAU) will be used as input for the 1D-TUB and ExoTIC model as well as to estimate the incoming GCR and SCR fluxes at the close-in exoplanets, respectively. (2) With the latter, the transport of CRs through the planetary magnetosphere is studied with PLANETOCOSMICS in order to provide TOA proton fluxes as input to model both the GCR- and SCR-induced atmospheric ionization as well as the resulting dose rates on the planetary surface. (3) The transport of CRs through the planetary magnetosphere is computed to provide TOA proton fluxes. (4) The secondary particle production due to $\mathrm{CR}$ interaction throughout the planetary atmosphere down to the surface is modeled. (5) The surface UVA, UVB, UVC exposure, and the radiation dose 


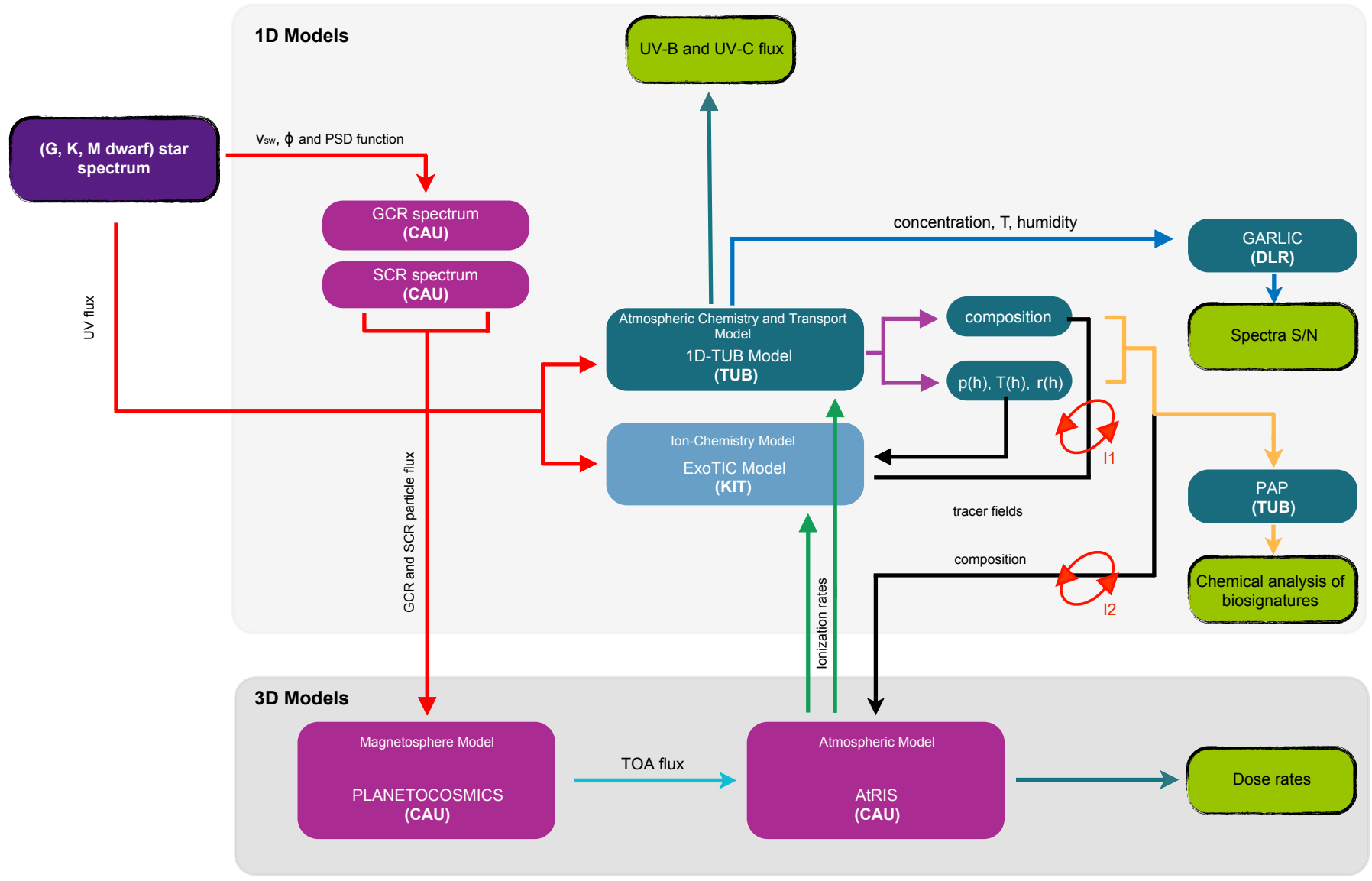

Fig. 3. Data exchange in the model suite and associated scientific goals. The following steps are performed: (1) (top left) measured stellar UV fluxes, the estimates of the stellar wind velocities $\left(v_{\mathrm{sw}}\right)$, stellar modulation $(\phi)$, and PSDs (Christian-Albrechts-Universität, hereafter CAU) are used as input for the 1D-TUB and ExoTIC models as well as to estimate the incoming GCR and SCR fluxes at the close-in exoplanets, respectively (highlighted by the red paths). (2) The transport of CRs through the planetary magnetosphere is studied with PLANETOCOSMICS in order to provide TOA proton fluxes as input to model both the GCR and SCR induced atmospheric ionization as well as the resulting dose rates on the planetary surface (light blue path), (3) computation of the secondary particle production due to CR interaction through the planetary atmosphere down to the surface, (4) calculation of the surface UV-A, UV-B and UV-C exposure as well as the radiation dose (turquoise paths), (5) determination of the impact of changing atmospheric ionization for the different atmospheric compositions as well as parameterization of the neutral atmosphere impact (green paths), (6) computation of the resulting atmospheric composition and climate (magenta paths), (7) performance of a pathway analysis in order to understand the biosignature chemical responses (orange paths), (8) utilization of the global atmospheric composition and temperature fields to compute atmospheric transit (primary) and emission (secondary) spectra (blue paths).

is computed. (6) The impact of changing atmospheric ionization for the different atmospheric compositions as well as parameterization of the neutral atmosphere impact is determined. (7) The resulting atmospheric composition and climate and especially the atmospheric neutral and ion chemistry of biosignatures (1D) for the different scenarios is computated. (8) A pathway analysis (varying in season and location) is performed in order to understand the biosignature chemical responses. (9) The global atmospheric composition and temperature fields are used to compute atmospheric transit (primary) and emission (secondary) spectra. (10) The 1D scenario between the different models for Earth-like conditions is validated. We note that two iterations within the framework are performed (red circles). Since the neutral species modeled with ExoTIC (KIT) strongly depend on the ionization rates provided by $\mathrm{CAU}$, iterations have to be performed (I1). After the first run, the computed neutral species are handed to TUB in order to determine the corresponding atmospheric composition, which is then provided as new input for AtRIS (I2). These two iterations are performed until no changes in neutral components and atmospheric composition due to the computed ionization rates occur.

\subsection{Testing the model suite on the (exo)planetary case-study of the modern-day Earth orbiting a G2V star}

This section studies the impact of GCRs and SCRs on the terrestrial atmosphere as an initial validation for our model-suite.

\subsubsection{The GCR and SCR environment at $1 \mathrm{AU}$}

As discussed in Sect. 2.1, the GCR flux at 1 AU strongly depends on the solar activity and can be described by the FFA (see Eq. (1)). Thereby, the only free parameter, the solar modulation parameter $\phi$, is directly proportional to the solar activity. PAMELA and AMS02 measurements suggest that typical modulation parameter values vary between 500 and 2000 MV. During quiet solar conditions, where the modulation effect is less pronounced, more low-energy GCR particles can be observed in the vicinity of Earth. Thus, solar minimum conditions are favored for use in investigating the upper limit of the GCR-induced atmospheric ionization. The quiescent GCR flux based on the LIS model by Herbst et al. (2017) is displayed as a solid black line in Fig. 4. 


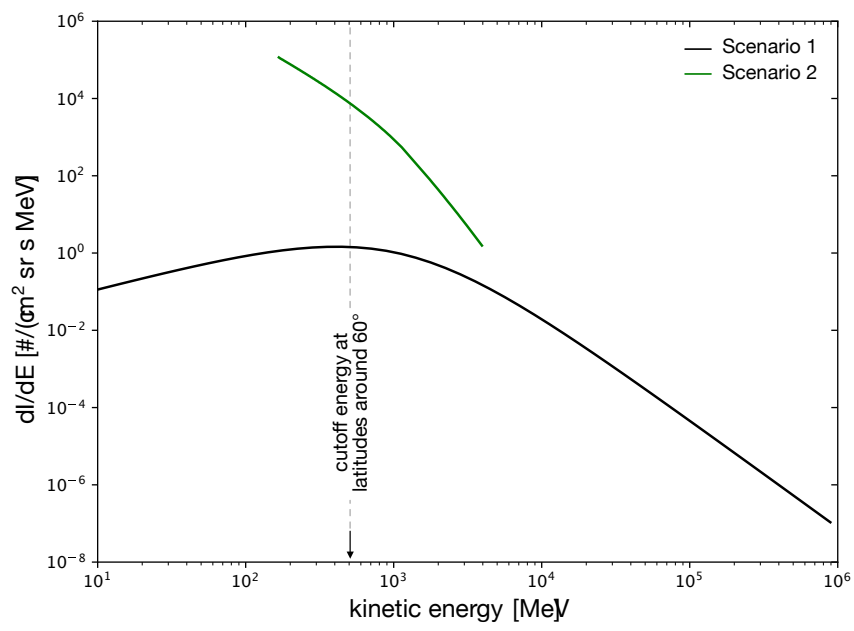

Fig. 4. Differential primary proton GCR spectrum during solar minimum (quiescent sun) conditions (scenario 1, black line) in comparison to the strongest ever measured ground-level enhancement (GLE) event (GLE05, February 23, 1956; scenario 2, green line). The gray dashed line indicates the cutoff energy, i.e., the energy a particle must have in order to reach a certain location within the terrestrial atmosphere at mid-latitudes around $60^{\circ}$.

In addition, the mean energy spectrum of the strongest GLE event ever measured during the Neutron Monitor era, GLE05 that occurred on February 23, 1956, is given as a solid green line. We note that the spectrum is based on the Band-Fit function proposed by Raukunen et al. (2018). Representing one of the strongest GLEs in solar history, this spectrum provides a good approximation for an upper limit of SEP flux. However, two things become apparent in Fig. 4: (i) solar particles with energies well above $1 \mathrm{GeV}$ are rare, and (ii) the differential proton intensities measured during such GLEs can be approximately one to more than four orders of magnitude higher than the quiescent GCR flux.

\subsubsection{The GCR- and SCR-induced atmospheric ionization, ion chemistry, and climate}

Cosmic rays penetrating the atmosphere of Earth mainly lose their energy due to ionization of ambient matter. Thereby, the probability of an interaction with the surrounding atmospheric gases drastically increases as the induced nucleonicelectromagnetic particle shower evolves towards the surface. Thus, the production of charged secondary particles also leads to the ionization of the middle as well as the lower atmosphere. This, however, strongly depends on (i) the energy of the primary particle, (ii) the secondary particle type, and the (iii) the atmospheric depth (see, e.g., Bazilevskaya et al. 2008; Usoskin et al. 2011; Banjac et al. 2019a).

Since GCRs are modulated due to the solar activity, the corresponding ionization is anti-correlated to the eleven-year solar cycle. In case of SEP events, however, only very highly energetic SCRs, such as the particles accelerated in strong SEP events, can induce and contribute significantly to the atmospheric ionization.

Numerically speaking, the CR-induced ionization rate $Q$ is given by

$Q\left(\phi, E_{\mathrm{C}}, x\right)=\sum_{i} \int_{E_{\mathrm{C}, i}}^{\infty} J_{i}(\phi, E) \cdot Y_{i}(E, x) \mathrm{d} E$,

depending on solar activity, geomagnetic location (here in form of the cutoff energy $E_{C}$ of a particle necessary in order to reach a certain location), and atmospheric depth $(x)$. Here, $i$ is the cosmic ray particle type, $J_{i}(\phi, E)$ the primary differential energy GCR or GLE spectrum, and $Y_{i}(E, x)$ the atmospheric ionization yield given by $Y_{i}(E, x)=\alpha \cdot \frac{1}{E_{\text {ion }}} \cdot \frac{\Delta E_{i}}{\Delta x}$, depending on the geometrical normalization factor $\alpha$ given by $2 \pi \int \cos (\theta) \sin \theta \mathrm{d} \theta$, the depth-dependent mean specific energy loss, and the atmospheric ionization energy $E_{\text {ion }}$. For the latter, most often an average ionization energy of $35 \mathrm{eV}$ (see Porter et al. 1976) is used. We note, however, that a study by Simon Wedlund et al. (2011) showed that this value strongly depends on the atmospheric composition, and that for modern Earth conditions a value of $32 \pm 1 \mathrm{eV}$ is more likely. This will be of great importance for the ionization of exoplanetary atmospheres with differing atmospheric compositions (see, e.g., discussion in Herbst et al. 2019b).

In the following studies, we assumed mid-latitudes around $60^{\circ}$. At such locations on Earth, the corresponding cutoff energy is in the order of $510 \mathrm{MeV}$ (indicated by the dashed vertical line in Fig. 4). Particles with energies below this threshold are not able to penetrate the geomagnetic field and cannot therefore contribute to the atmospheric ionization. The computed ionization rates have a strong influence on the computation of atmospheric neutral species and hence on the atmospheric composition (see discussion below). Ionization rates are computed using the initial climate and composition data from the 1D-TUB model ("Modern Earth Atmosphere", TUB). These rates are then implemented in both the 1D-TUB and ExoTIC models to provide an event-based set of atmospheric neutral species and atmospheric compositions.

As discussed in Sect. 5, it is crucial to reach stable neutral component and atmospheric composition conditions. In order to achieve those, multiple iteration rounds between the different models (1D-TUB, AtRIS, and ExoTIC) may be necessary.

In this study, we investigate two different scenarios:

- the influence of the quiescent Sun $(\phi=550 \mathrm{MV})$, only taking into account GCRs: scenario 1;

- the influence of the Febrary 23, 1956, GLE (GLE05), one of the strongest ever measured: scenario 2 .

In the case of scenario 1 , only one iteration between the three models was necessary, whereas for scenario 2 more iterations were needed. The different iteration-dependent results of scenario 2 are shown in Fig. 5. Here, the corresponding temperature profile (upper left panel), as well as the molecular profiles of $\mathrm{O}_{3}$ (upper right panel), $\mathrm{CH}_{4}$ (middle left panel), and $\mathrm{N}_{2} \mathrm{O}$ (middle right panel) provided via the 1D-TUB model, are given. As mentioned previously, the atmospheric compositions of the initial run (solid black lines) are based on scenario 1. Implemented into AtRIS and induced to a much harsher SCR environment, the resulting ionization rate profiles (see bottom panel) that are provided as input for the ExoTIC model will lead to changes of the initial atmospheric profiles of the 1D-TUB model. In total, three iterations between the models were required in order to achieve a stable neutral component and atmospheric composition conditions. As can be seen, the initial conditions (solid black line) strongly differ from the final iteration conditions (red lines). Moreover, comparing the final temperature profiles that reproduce the characteristic temperature-layered regions of Earth (upper left panel) for scenario 1 (black solid line) with those of scenario 2 suggests that the profiles are similar at altitudes below $\sim 10 \mathrm{~km}$, while differences of up to $10 \mathrm{~K}$ can be observed at higher altitudes. Thereby, the temperatures are lower up to an altitude of $\sim 45 \mathrm{~km}$, while being slightly higher at higher altitudes due to increased $\mathrm{O}_{3}$ abundances (see also Fig. 13). 

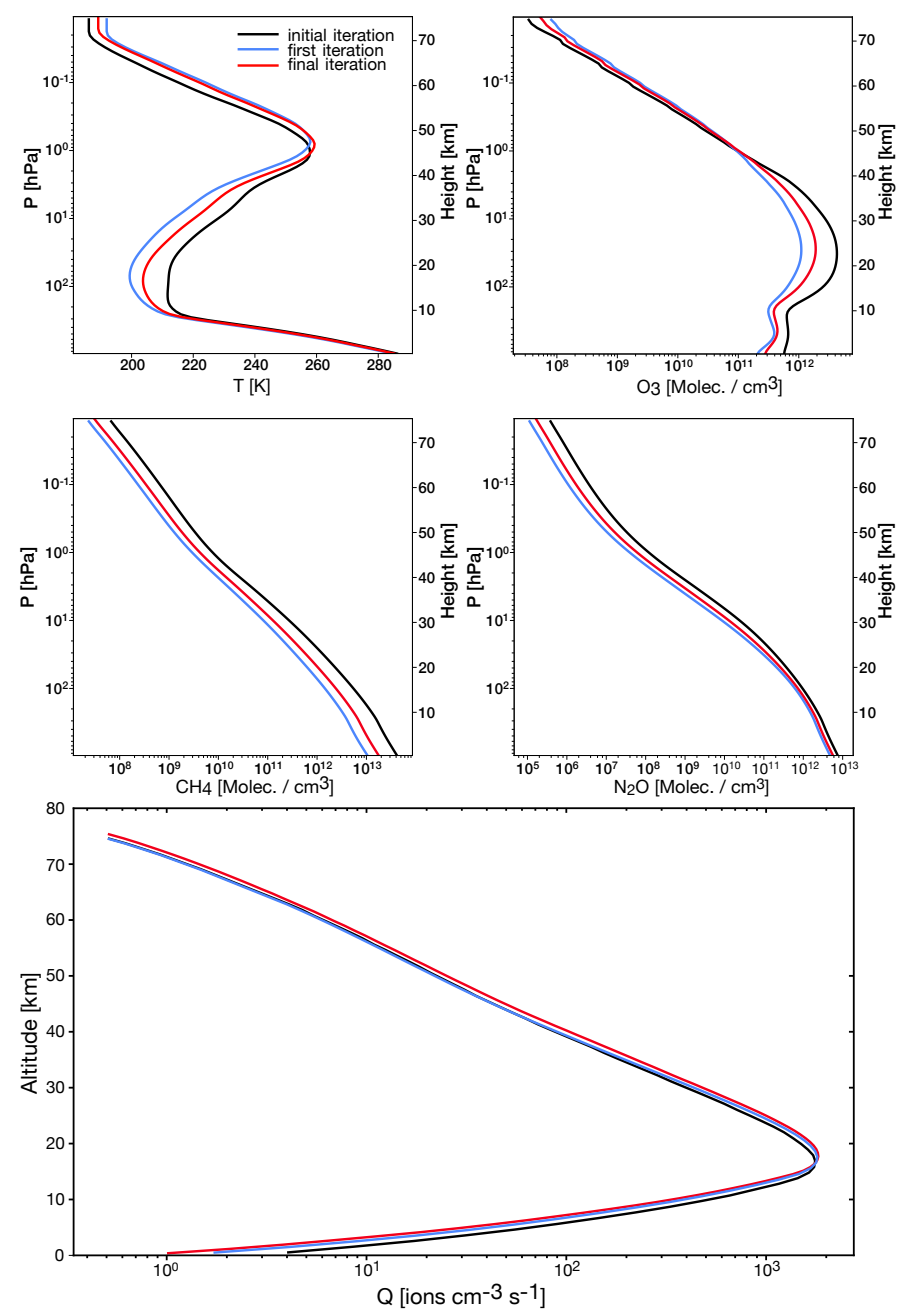

Fig. 5. Model results for scenario 2 (GLE05). Three simulation rounds were required in order to achieve converged output. Here, the initial conditions are displayed in black, while the first and second iterations are shown in blue and red, respectively. We note that the results of the second iteration were used in a third iteration. Since the results of the third iteration are similar to those of the second, they are not shown here. We note further that the initial conditions (solid black line) are the same as the final conditions of scenario 1 .

In addition, the climate model discussed in Sect. 4.4.1 featured an input surface albedo value, which was adjusted step-by-step until the observed global mean temperature for modern Earth equal to $288 \mathrm{~K}$ was reached. This is a rather common procedure in the Earth-like literature (Segura et al. 2003) and is equivalent to placing an artificial reflecting "cloud" layer at the surface which is intended to compensate straightforwardly for the lack of cloud microphysics in the model. The surface albedo value in the model $(=0.232)$ is accordingly somewhat higher than the global mean observed value of 0.15 (Hummel \& Reck 1979). The convective and radiative modules could successfully reproduce the main features of the global mean temperature profile of modern Earth, including the tropospheric lapse rate, the magnitude of the cold trap, and the temperature inversion in the middle atmosphere due to heating from ozone absorption.

The chemical network discussed in Sect. 4.4.2 calculates a stationary, global-mean ozone column of 313.5 Dobson Units for modern Earth conditions. This compares well with observations in the atmosphere of modern Earth (WMO 1994). Ozone features
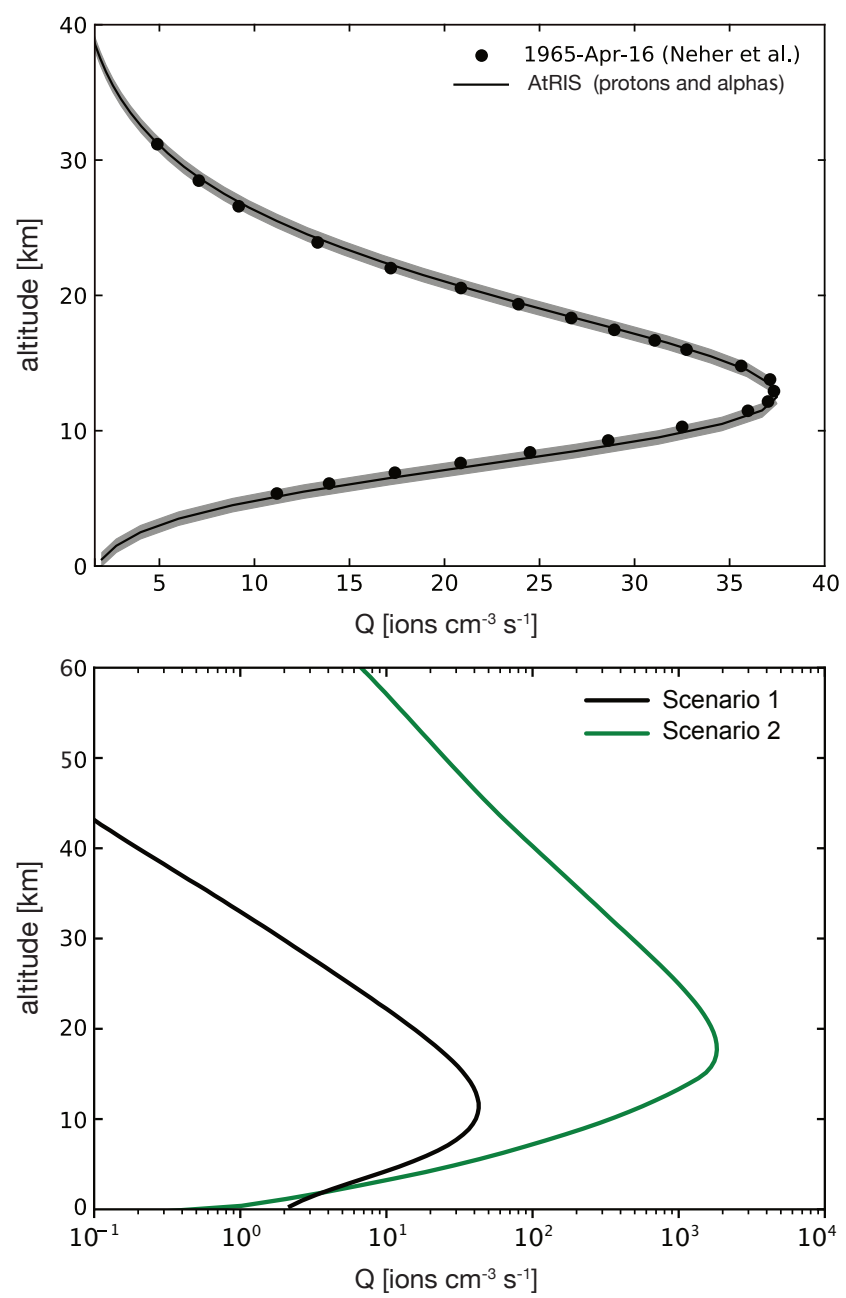

Fig. 6. Upper panel: validation of the modeled GCR-induced ion-pair production rates (solid line) against balloon measurements performed by Neher (1971) (dots, see Banjac et al. 2019a, for further validation). Lower panel: CR-induced atmospheric ionization for scenario 1 (quiescent Sun) and scenario 2 (GLE05) displayed as black and green curves, respectively. As can be seen, differences of up to three orders of magnitude occur.

rather complex photochemical-climate responses, and therefore the reproduction of its global column is a good performance test of the chemistry module. Long-lived source gases such as carbon dioxide were set to 1990 levels at the surface (=355 ppm) consistent with previous model studies of Earth-like atmospheres (see, e.g., Segura et al. 2003). Surface concentrations of biomass and related gases such as methane, chloromethane, nitrous oxide, and carbon monoxide were also fixed to modern values. The corresponding global mean mass fluxes required to maintain these surface concentrations compared reasonably well with modern Earth observations (see, e.g., Grenfell et al. 2014, for more details on the procedure).

Figure 6 shows our modeled ionization rate profiles. Here, the upper panel shows a comparison between the altitudedependent ionization profile measured during a balloon flight on April 16, 1965 (black dots Neher 1971), and the model results of AtRIS (black line). The figure suggests good agreement between simulations and the measurement. A comparison of the scenario-based stable-condition ionization rate profiles is given in the lower panel of Fig. 6. As can be seen, the strong SEP event of scenario 2 (green curve) leads to drastically increased 

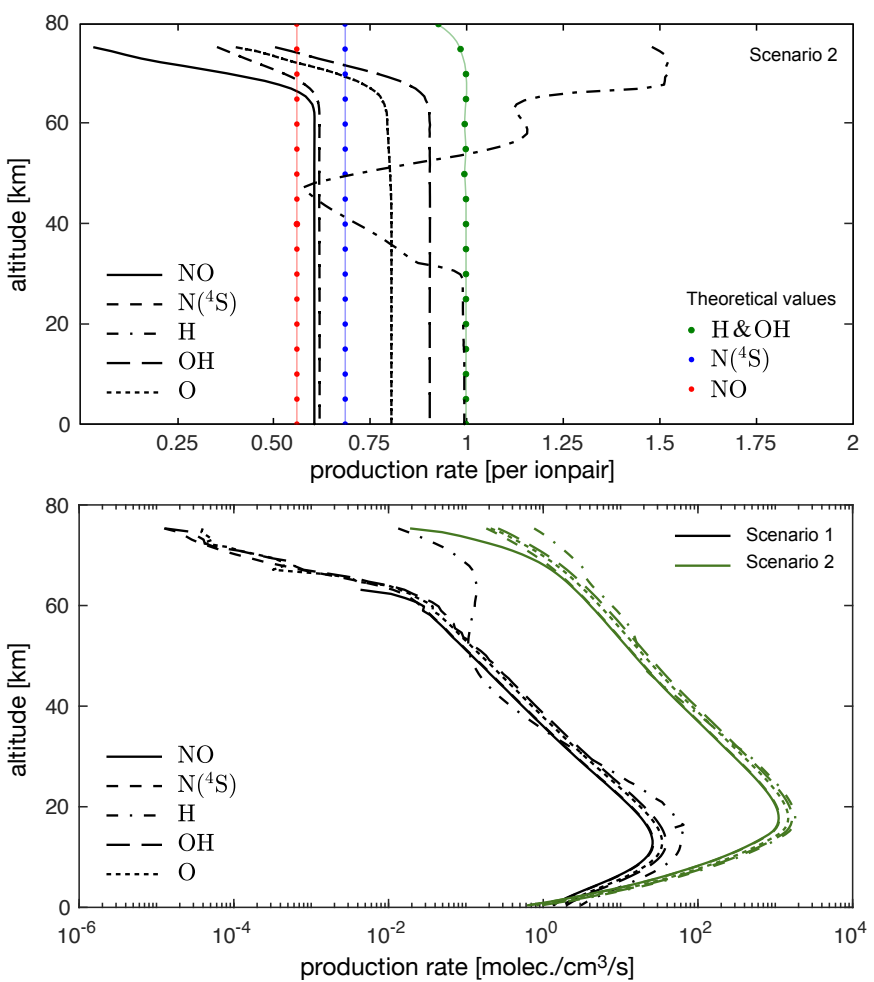

Fig. 7. Upper panel: production rates of neutral species $\mathrm{NO}, \mathrm{N}\left({ }^{4} \mathrm{~S}\right)$, $\mathrm{H}, \mathrm{OH}$ and $\mathrm{O}$ due to ion-chemistry processes calculated with ExoTIC (black lines) for scenario 2 (GLE05) compared to theoretical values of existing parameterizations (coloured dots, see text). Lower panel: production rates of $\mathrm{NO}, \mathrm{N}\left({ }^{4} \mathrm{~S}\right), \mathrm{H}, \mathrm{OH}$ and $\mathrm{O}$ due to ion-chemistry processes in the atmosphere for scenario 1 (quiescent Sun, black lines) and scenario 2 (GLE05, green lines).

altitude-dependent ionization rates at all altitudes compared to the results of scenario 1 (black curve). Differences of up to three orders of magnitude can be found. These are also reflected in the production rates of neutral species, as can be seen in Fig. 7.

As described in Sect. 4.3, ion chemistry processes influence the concentrations of the neutral constituents of the atmosphere. For the two scenarios discussed here, this mainly affects NO, $\mathrm{N}\left({ }^{4} \mathrm{~S}\right), \mathrm{H}, \mathrm{OH}$, and $\mathrm{H}_{2} \mathrm{O}$. The upper panel of Fig. 7 shows a comparison of the production rates derived with the full ion chemistry calculation using ExoTIC with simple parameterization models as summarized in Jackman et al. (2005b). The parameterizations consider the terrestrial homosphere with a fixed mixing ratio of $\mathrm{N}_{2}$ and $\mathrm{O}_{2}$. The parameterization for $\mathrm{N}\left({ }^{4} \mathrm{~S}\right)$ and NO is originally based on Porter et al. (1976). It only considers the direct impact of dissociation and dissociative ionization; no ion chemistry is considered. This leads to a strong deviation above $\approx 70 \mathrm{~km}$, where the impact of ion chemistry becomes more important and where the primary ions vary due to the continuing photolysis of $\mathrm{O}_{2}$ (see, e.g., Nieder et al. 2014). Below this altitude, production rates of $\mathrm{N}\left({ }^{4} \mathrm{~S}\right)$ and $\mathrm{NO}$ are constant functions of the ion pair production rate, though the factors differ because ExoTIC also considers the formation of NO by ion chemistry, leading to a higher formation rate of NO than in the parameterization. The parameterization of $\mathrm{H}$ and $\mathrm{OH}$ is based on Solomon \& Crutzen (1981) and considers a simplified set of protonized cluster ion reactions for the homosphere and a fixed water-vapor profile. It also deviates strongly from the ExoTIC values above $70 \mathrm{~km}$ altitude presumably because different water vapor profiles are used, and due to the more complex ion chemistry considered in ExoTIC. Below this altitude, the rate of $\mathrm{OH}$ production is about $10 \%$ lower in ExoTIC than in the parameterization. This has already been discussed in Sinnhuber et al. (2012), and is due to the formation of protonized water cluster ions via $\mathrm{NO}^{+}\left(\mathrm{H}_{2} \mathrm{O}\right)_{3}+\mathrm{H}_{2} \mathrm{O}$, which do not release $\mathrm{OH}$. In the altitude range $40-60 \mathrm{~km}$, the production rate of $\mathrm{H}$ as calculated by ExoTIC varies with altitude, deviating strongly from the parameterization values. In this altitude region, a transition occurs from recombination of the protonized water cluster ions by negative ions to recombination by electrons (not shown). Apparently, this transition is not smooth, leading to the observed dip in the $\mathrm{H}$ production. These differences between results from ExoTIC considering full ion chemistry to parameterizations considering the homosphere (either with or without simplified ion chemistry) emphasize the importance of ion chemistry; for exoplanets with atmospheric compositions widely different from that of the terrestrial atmosphere, it is mandatory to explicitly consider the formation of primary ions and the subsequent effects of ion chemistry upon the neutral constituents. The lower panel of Fig. 7 displays the modeled production rates of these species due to ion-chemistry processes in the atmosphere for the final iteration based on the inputs from the 1D-TUB model and the ionization rates provided by AtRIS. Here, the production rates for scenario 1 are displayed in black, those of scenario 2 in green.

\section{Impact of cosmic rays on biosignatures as well as the surface UV-radiation and radiation dose}

Since the CR-induced atmospheric ion-pairs have a direct influence on the atmospheric radiation dose, the atmospheric chemistry and, thus, on potential biosignatures like $\mathrm{O}_{3}, \mathrm{~N}_{2} \mathrm{O}$, or $\mathrm{CH}_{4}$, the results of Fig. 6 as discussed in the following section are used as input to model the influence on the planetary atmosphere, addressing crucial questions such as: How do CRs and the resulting photochemistry affect the abundance of potential biosignatures? What is the radiation dose at the surface of Earth and Earth-like planets and how strong is the surface UV radiation exposure? How do CRs affect the spectral features of biosignatures?

\subsection{How do CRs and the resulting photo-chemistry affect the abundance of potential biosignatures?}

The panels of Figs. 8 and 9 show the contribution of ozone loss cycles and ozone production cycles for scenarios 1 (left panels) and 2 (right panels) as a function of atmospheric pressure and altitude, respectively. These results were obtained with PAP (Lehmann 2004) which analyses the chemical output from the 1D-TUB model.

The results in Fig. 8 illustrate the well-studied ozone-UV feedback, a "self-healing" mechanism (negative feedback, see, e.g., WMO 1994) which helps to maintain the ozone layer and operates as follows: processes which reduce ozone, for example due to $\mathrm{NO}_{x}$ and $\mathrm{HO}_{x}$ production from CRs, lead to stronger penetration of UV into the layers immediately below. Here, they result in stimulated oxygen photolysis, and therefore enhanced ozone formation.

As can be seen, the peak loss rates for ozone pathways increase by $15 \%$, that is, from $4.6 \times 10^{6}$ molecules $\mathrm{cm}^{-3} \mathrm{~s}^{-1}$ in scenario 1 up to $5.3 \times 10^{6}$ molecules $\mathrm{cm}^{-3} \mathrm{~s}^{-1}$ in scenario 2 . Additionally, the altitude corresponding to the peak loss rates moves downwards from $43.5 \mathrm{~km}$ in scenario 1 to $40.5 \mathrm{~km}$ in scenario 2. For the quiescent scenario, $\mathrm{NO}_{x}$ cycles are important in the low to mid stratosphere, whereas $\mathrm{HO}_{x}$ and $\mathrm{O}_{x}$ cycles 

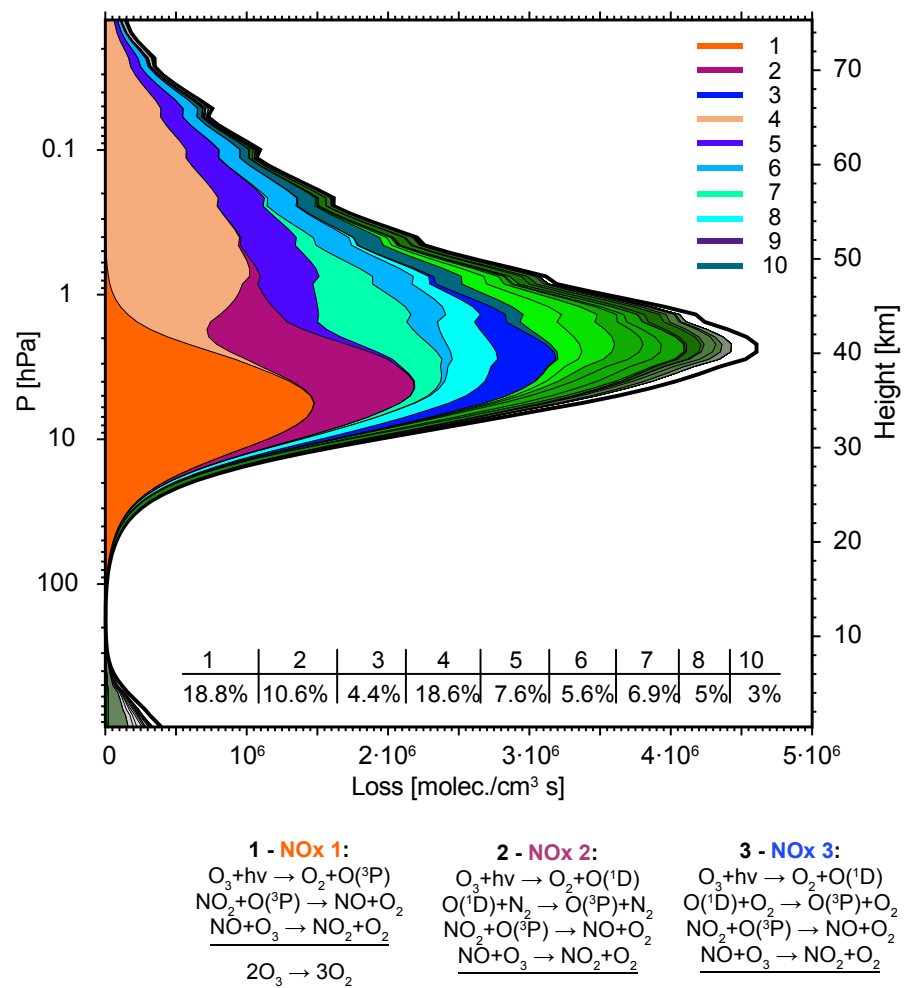

$$
\begin{aligned}
& 2 \text { - NOx 2: } \\
& \text { 3- }-\mathrm{NOx} 3: \\
& \mathrm{O}\left({ }^{\prime} \mathrm{D}\right)+\mathrm{N}_{2} \rightarrow \mathrm{O}\left({ }^{3} \mathrm{P}\right)+\mathrm{N}_{2} \quad \mathrm{O}\left({ }^{3} \mathrm{D}\right)+\mathrm{O}_{2} \rightarrow \mathrm{O}\left({ }^{3} \mathrm{P}\right)+\mathrm{O}_{2} \\
& \mathrm{NO}_{2}+\mathrm{O}\left({ }^{3} \mathrm{P}\right) \rightarrow \mathrm{NO}+\mathrm{O}_{2} \quad \mathrm{NO}_{2}+\mathrm{O}\left({ }^{3} \mathrm{P}\right) \rightarrow \mathrm{NO}+\mathrm{O}_{2} \\
& {\mathrm{NO}+\mathrm{O}_{3} \rightarrow \mathrm{NO}_{2}+\mathrm{O}_{2} \quad \mathrm{NO}+\mathrm{O}_{3} \rightarrow \mathrm{NO}_{2}+\mathrm{O}_{2}} \\
& 2 \mathrm{O}_{3} \rightarrow 3 \mathrm{O}_{2} \\
& 7 \text { - Ox 1: } \\
& \mathrm{O}_{3}+\mathrm{hv} \rightarrow \mathrm{O}_{2}+\mathrm{O}\left({ }^{1} \mathrm{D}\right) \\
& \mathrm{O}\left({ }^{3} \mathrm{D}\right)+\mathrm{N}_{2} \rightarrow \mathrm{O}\left({ }^{3} \mathrm{P}\right)+\mathrm{N}_{2} \\
& \mathrm{O}\left({ }^{3} \mathrm{P}\right)+\mathrm{O}_{3} \rightarrow \mathrm{O}_{2}+\mathrm{O}_{2} \\
& 2 \mathrm{O}_{3} \rightarrow 3 \mathrm{O}_{2} \\
& 2 \mathrm{O}_{3} \rightarrow 3 \mathrm{O}_{2} \\
& 8 \text { - } 0 \times 2 \text { : } \\
& \mathrm{O}_{3}+\mathrm{hv} \rightarrow \mathrm{O}_{2}+\mathrm{O}\left({ }^{3} \mathrm{P}\right) \\
& \mathrm{O}\left({ }^{3} \mathrm{P}\right)+\mathrm{O}_{3} \rightarrow \mathrm{O}_{2}+\mathrm{O}_{2} \\
& 2 \mathrm{O}_{3} \rightarrow 3 \mathrm{O}_{2}
\end{aligned}
$$

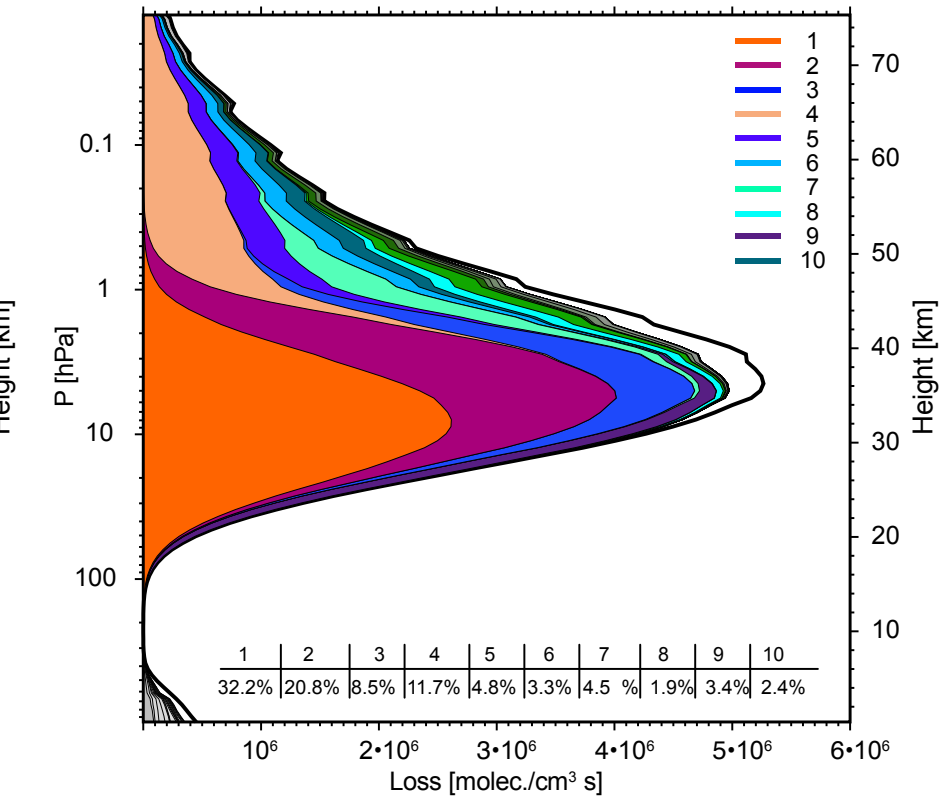

$$
\begin{gathered}
4-\mathrm{HO} \times 1: \\
\mathrm{O}_{3}+\mathrm{hv} \rightarrow \mathrm{O}_{2}+\mathrm{O}\left({ }^{1} \mathrm{D}\right) \\
\mathrm{O}\left({ }^{1} \mathrm{D}\right)+\mathrm{N}_{2} \rightarrow \mathrm{O}\left({ }^{3} \mathrm{P}\right)+\mathrm{N}_{2} \\
\left.\mathrm{HO}+\mathrm{O}^{(} \mathrm{P}\right) \rightarrow \mathrm{OH}+\mathrm{O}_{2} \\
\mathrm{OH}+\mathrm{O}\left({ }^{3} \mathrm{P}\right) \rightarrow \mathrm{H}+\mathrm{O}_{2} \\
\mathrm{H}+\mathrm{O}_{2}+\mathrm{M} \rightarrow \mathrm{HO}_{2}+\mathrm{M} \\
2 \mathrm{O}_{3} \rightarrow 3 \mathrm{O}_{2}
\end{gathered}
$$

5 - HOx 2: $\mathrm{O}_{3}+\mathrm{hv} \rightarrow \mathrm{O}_{2}+\mathrm{O}\left({ }^{1} \mathrm{D}\right)$ $\mathrm{O}\left({ }^{3} \mathrm{D}\right)+\mathrm{O}_{2} \rightarrow \mathrm{O}\left({ }^{3} \mathrm{P}\right)+\mathrm{O}_{2}$ $\left.\mathrm{HO}^{+}+\mathrm{O}^{2} \mathrm{P}\right) \rightarrow \mathrm{OH}+\mathrm{O}_{2}$ $\mathrm{OH}+\mathrm{O}\left({ }^{3} \mathrm{P}\right) \rightarrow \mathrm{H}+\mathrm{O}_{2}$ $\mathrm{H}+\mathrm{O}_{2}+\mathrm{M} \rightarrow \mathrm{HO}_{2}+\mathrm{M}^{2}$

$$
2 \mathrm{O}_{3} \rightarrow 3 \mathrm{O}_{2}
$$

$$
6 \text { - HOx 3: }
$$

$\mathrm{O}_{3}+\mathrm{hv} \rightarrow \mathrm{O}_{2}+\mathrm{O}\left({ }^{3} \mathrm{P}\right)$

$\mathrm{HO}_{2}+\mathrm{O}\left({ }^{3} \mathrm{P}\right) \stackrel{\mathrm{OH}}{\rightarrow}+\mathrm{O}_{2}$ $\mathrm{OH}+\mathrm{O}\left({ }^{3} \mathrm{P}\right) \rightarrow \mathrm{H}+\mathrm{O}_{2}$

9 - NO 1:

10 - HOx 4:

$\mathrm{NO}_{2}+\mathrm{hv} \rightarrow \mathrm{NO}+\mathrm{O}\left({ }^{3} \mathrm{P}\right) \quad \mathrm{O}_{3}+\mathrm{hv} \rightarrow \mathrm{O}_{2}+\mathrm{O}\left({ }^{1} \mathrm{D}\right)$

$2 \cdot\left(\mathrm{NO}+\mathrm{O}_{3} \rightarrow \mathrm{NO}_{2}+\mathrm{O}_{2}\right) \quad \mathrm{O}\left({ }^{3} \mathrm{D}\right)+\mathrm{N}_{2} \rightarrow \mathrm{O}\left({ }^{3} \mathrm{P}\right)+\mathrm{N}_{2}$

$\left.\mathrm{NO}_{2}+\mathrm{O}\left({ }^{3} \mathrm{P}\right) \rightarrow \mathrm{NO}+\mathrm{O}_{2} \quad \mathrm{OH}+\mathrm{O}^{2} \mathrm{P}\right) \rightarrow \mathrm{H}+\mathrm{O}_{2}$

$$
2 \mathrm{O}_{3} \rightarrow 3 \mathrm{O}_{2} \quad \frac{\mathrm{H}+\mathrm{O}_{3} \rightarrow \mathrm{OH}+\mathrm{O}_{2}}{2 \mathrm{O}_{3} \rightarrow 3 \mathrm{O}_{2}}
$$

Fig. 8. Ozone loss cycles determined by PAP corresponding to scenario 1 (quiescent Sun, left panel) and scenario 2 (GLE05, right panel) are shown on a linear scale from the surface to the mid mesosphere $(\sim 75 \mathrm{~km})$. Please note that the figures show all pathways with a percentage contribution above $0.01 \%$ and that only the ten most important pathways are tabulated in the subpanel.

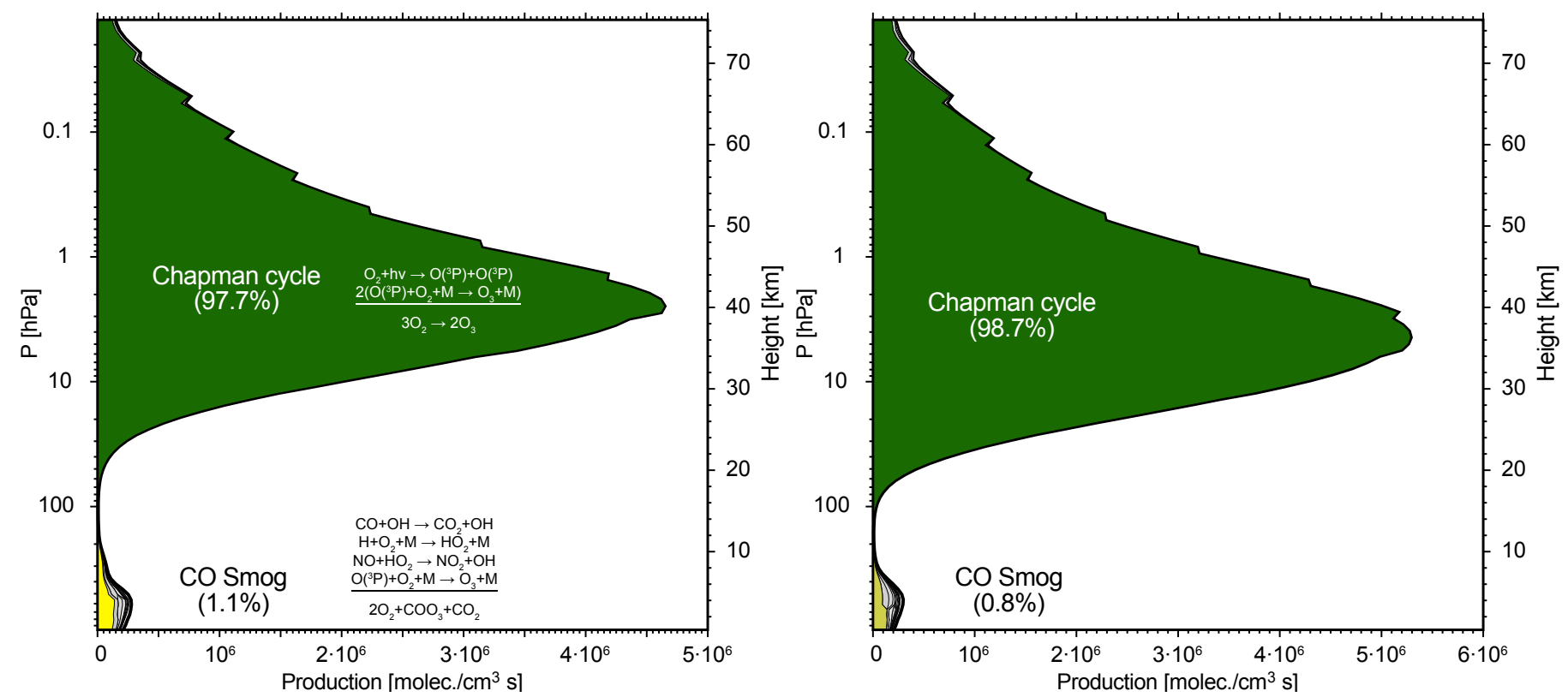

Fig. 9. Left panel: ozone production cycles determined by PAP for scenario 1 (quiescent Sun). Right panel: ozone production cycles determined by PAP for scenario 2 (GLE05). 


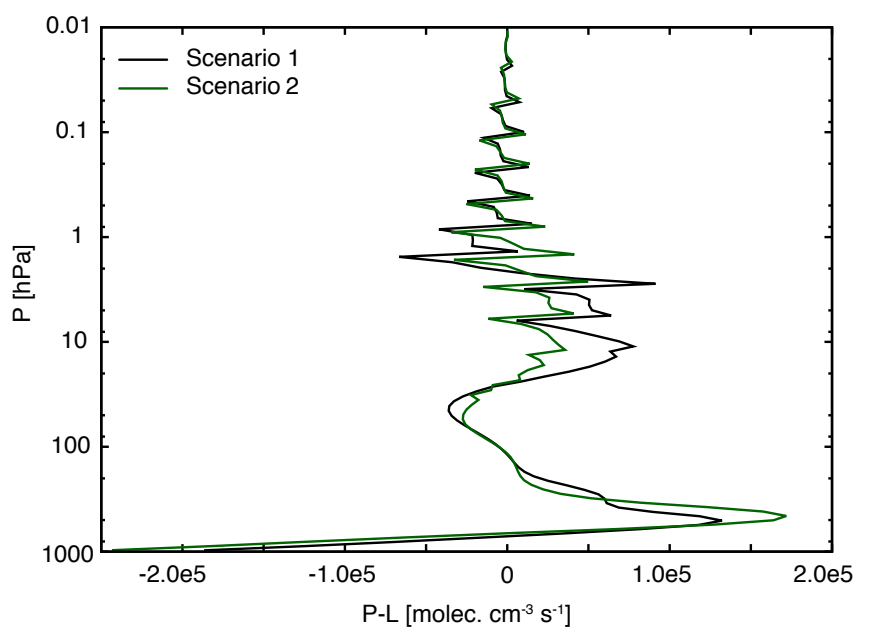

Fig. 10. Production $(\mathrm{P})$ minus loss $(\mathrm{L})$ of gas-phase ozone $\left(\mathrm{O}_{3}\right)$ for scenario 1 (quiescent Sun, black line) and scenario 2 (GLE05, green line) vs. pressure $(\mathrm{hPa})$.

dominate the mid to upper stratosphere. In scenario 2, three $\mathrm{NO}_{x}$-cycles are stimulated by the interacting cosmic rays and they dominate $\mathrm{NO}_{x}$-loss pathways for ozone in the low to mid stratosphere. We note that some lines show a sawtooth-type pattern, especially in the upper atmosphere which is related to an interpolation between the different vertical grids of the climate and chemistry modules.

The apparent increase of $\mathrm{NO}_{x}$ in the right (scenario 2) compared with the left panel (scenario 1) of Fig. 8 leads to a reduction of ozone (as can also be seen in Fig. 13). Therefore, a deeper penetration of UV occurs, which shifts the maximum of the ozone production to lower altitudes. The ozone production by the "CO smog cycle" in the troposphere also increases by a factor of two during the flaring scenario.

We note that we required PAP to connect partial pathways producing $\mathrm{O}\left({ }^{3} \mathrm{P}\right)$ with partial pathways consuming $\mathrm{O}\left({ }^{3} \mathrm{P}\right)$ at all altitudes, that is including above $60 \mathrm{~km}$, although the lifetime of $\mathrm{O}\left({ }^{3} \mathrm{P}\right)$ is longer than that of ozone there. Without this specification, the PAP does not build a Chapman-pathway above $60 \mathrm{~km}$ and instead outputs the single reaction: $\mathrm{O}\left({ }^{3} \mathrm{P}\right)+\mathrm{O}_{2}+\mathrm{M} \rightarrow$ $\mathrm{O}_{3}+\mathrm{M}$ as the main $\mathrm{O}_{3}$ source (see, e.g., Fig. 6a in Grenfell et al. 2013a).

Figure 10 shows the net production (production minus loss, $\mathrm{P}-\mathrm{L}$ ) of ozone due to the changes in atmospheric chemistry. The results suggest that scenario 2 (GLE case, green line) is shifted to the left of the modern quiescent Earth case (black line) in the heart of the ozone layer at around $10 \mathrm{hPa}(\sim 30 \mathrm{~km})$. This is consistent with ozone loss due to $\mathrm{NO}_{x}, \mathrm{O}_{x}$, and $\mathrm{HO}_{x}$ cycles in scenario 2 displayed in Fig. 8. In the upper troposphere between 200 and $500 \mathrm{hPa}$, the results of scenario 2 (green line) are shifted to the right compared with the results of scenario 1 , which is consistent with ozone production due to the smog cycle (favored by increased $\mathrm{NO}_{x}$ ).

The rates of the $\mathrm{NO}_{x}\left(=\mathrm{NO}+\mathrm{NO}_{2}\right)$ cycles presented in Fig. 8 show the combined effect of increased $\mathrm{NO}_{x}$ and decreased ozone concentrations (scenario 2 vs. scenario 1 ). In order to demonstrate the effect of changes of $\mathrm{NO}_{x}$ on ozone more clearly, we calculated pseudo first-order loss rate coefficients of ozone. For this, we assume that some process, for example a catalytic cycle, removes ozone at a rate $R$ :

$\frac{\delta\left[\mathrm{O}_{3}\right]}{\delta t}=-R$
Table 1. Pseudo first-order loss rate for both scenarios at $34.6 \mathrm{~km}$ (see text).

\begin{tabular}{cccc}
\hline \hline Scenario & $\rho_{\text {total }}\left(\right.$ molec cm $\left.^{-3}\right)$ & $\mathrm{O}_{3}(\mathrm{ppm})$ & Scaled rate \\
\hline 1 & $1.73 \times 10^{17}$ & 9.44 & $9.05 \times 10^{-7}$ \\
2 & $1.71 \times 10^{17}$ & 5.01 & $2.86 \times 10^{-6}$ \\
\hline
\end{tabular}

Notes. $\rho_{\text {total }}$ represents the total air density and the scaled rates are given by $R /\left(\mathrm{O}_{3} \cdot \rho_{\text {total }}\right)$.

A simple reformulation of this equation yields

$\frac{\delta\left[\mathrm{O}_{3}\right]}{\delta t}=-K \cdot\left[\mathrm{O}_{3}\right]$,

where the pseudo first-order loss rate coefficient,

$K=\frac{R}{\left[\mathrm{O}_{3}\right]}$,

expresses how fast ozone is removed by the process under consideration. Table 1 shows the pseudo first-order loss rate coefficient of ozone with respect to the first $\mathrm{NO}_{x}$ cycle $\left(\mathrm{NO}_{x} 1\right)$ at $34.6 \mathrm{~km}$ where it has its maximum contribution (Fig. 8). The scaled rates in Table 1 (which are taken from the PAP analysis to be the mass flux through the $\mathrm{NO}_{x} 1$ cycle) suggest that this cycle destroys ozone faster (in this case by a factor 3.2) in scenario 2 than in scenario 1. Similar results have been found for the other $\mathrm{NO}_{x}$ cycles (not shown here).

\subsection{What is the radiation dose at the surface of (exo)planet Earth and how strong is the surface UV radiation exposure?}

Strong solar particle events, in particular those that alter the radiation field near the planetary surface (GLEs), lead to an additional ionization as well as production of secondary particles throughout the entire atmosphere (see also Fig. 6). Recently, a new feature of AtRIS was developed that, for the first time, directly enables parametric studies of the interrelation of incident radiation and the resulting absorbed dose throughout the atmosphere (see Banjac et al. 2019b). Using their approach, we computed the altitude-dependent absorbed and equivalent dose rates for both scenarios. The results of this study are shown in the upper panels of Fig. 11. Here the absorbed dose rates are displayed on the left, while the equivalent dose rates are given in the right panel. As can be seen, a strong GLE event (green lines) such as the one on February 23, 1956, has a strong impact on both quantities throughout all atmospheric layers. During this latter event, the surface absorbed dose rate increases by a factor of two, while at altitudes above $20 \mathrm{~km}$ a relative increase of almost two orders of magnitude becomes evident.

Moreover, the lower panels of Fig. 11 show the effect of the CR-induced chemical changes on the atmospheric UV environment, which are a direct output of the 1D-TUB model. As can be seen, neither UVA nor UVC show any significant differences between the quiescent case with GCR background (black) and the flaring case (green). The UVB environment, on the contrary, shows a clear increase in the lower atmosphere. In our Earthlike atmosphere, the main absorber in the UVB range is $\mathrm{O}_{3}$. The enhanced UVB environment shown here corresponds well with the reduction of $\mathrm{O}_{3}$ by up to $50 \%$ for the flaring case, as shown in Figs. 12 and 13. 

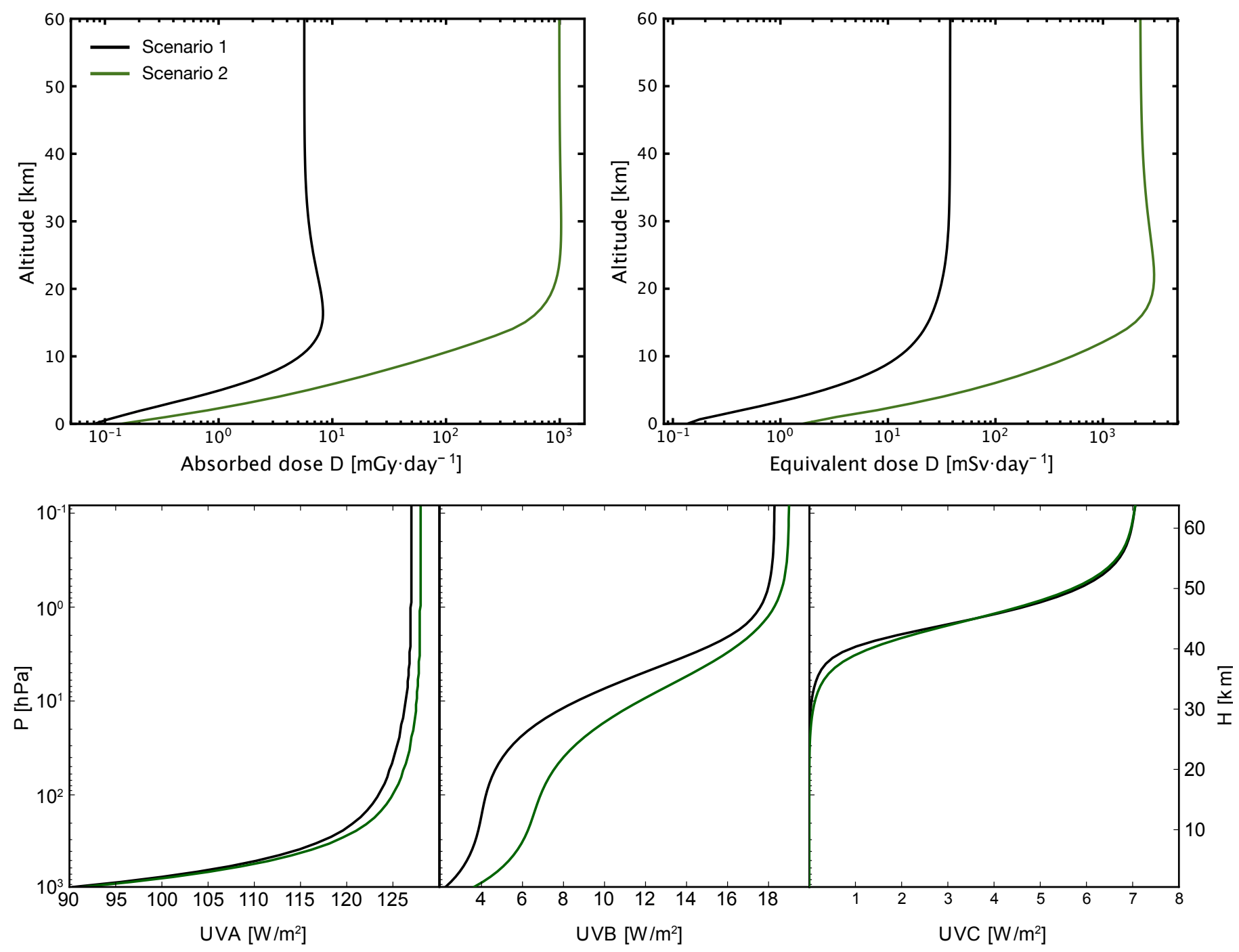

Fig. 11. Upper panels: computed absorbed (left panel) and equivalent (right panel) dose rates during scenario 1 (quiescent Sun, black lines) and scenario 2 (GLE05, green lines). Lower panels: atmospheric profiles of the UVA (left), UVB (middle), and UVC (right) environments according to both scenarios.

As a direct consequence, during a solar event as strong as that of February 1956, a harmful UVB radiation of about $3.5 \mathrm{~W} \mathrm{~m}^{-2}$ would result at the planetary surface.

\subsection{How do CRs affect the spectral features of biosignatures?}

Figure 12 shows the modeled transmission spectra derived with the GARLIC model for the two scenarios based on the finaliteration input of (T, $p$, composition) from the 1D-TUB model. The calculated wavelength range extends from 0.3 to $30 \mu \mathrm{m}$, corresponding to one of the modes of the NIR-Spec infrared spectrometer aboard the upcoming JWST mission. Characteristic spectral features of the modern Earth are seen. For example, the Rayleigh extinction "slope" in the UV-visible, the rotationalvibrational water, and methane bands in the NIR and the characteristic ozone and carbon dioxide fundamentals at 9.6 and $15 \mu \mathrm{m}$, respectively. Weaker spectral features of proposed atmospheric biosignatures such as $\mathrm{O}_{2}$ (in the visible) and $\mathrm{N}_{2} \mathrm{O}$ (at around $7.8 \mu \mathrm{m}$ ) are also apparent. A comparison between the two scenarios shows moderate suppression of some spectral features for the GLE event (green lines); for example, for $\mathrm{O}_{3}(9.6 \mu \mathrm{m})$ and
$\mathrm{CH}_{4}$ (NIR) due to for example photochemical loss induced by cosmic rays. In addition, Tabataba-Vakili et al. (2016) proposed an $\mathrm{HNO}_{3}$ feature around $12 \mu \mathrm{m}$ as a CR signature for Earth-like planets around active M-stars. The fact that we see the same feature during a strong GLE in the atmosphere of Earth (see Fig. 12) strengthens their argument. However, Scheucher et al. (2018) recently showed that these results strongly depend on the strength of the event as well as the atmospheric composition of the exoplanetary atmosphere.

Although the GLE event of February 1956 was rather modest compared to what has been observed for flaring M-dwarfs like AD Leonis or Proxima Centari (see, e.g., Herbst et al. 2019a), its impact on the atmospheric biosignatures is clearly visible. For a better overview of the differences in the transit spectra, Fig. 13 displays the abundance changes for the molecules $\mathrm{O}_{3}$ (upper left panel), $\mathrm{N}_{2} \mathrm{O}$ (upper right panel), $\mathrm{CH}_{4}$ (lower left panel), and $\mathrm{CH}_{3} \mathrm{Cl}$ (lower right panel): these molecules, in particular, are known on Earth to be heavily influenced by life. In the case of $\mathrm{O}_{3}$, our results show decreases of up to $50 \%$ in the lower-mid stratosphere as well as the lower troposphere for the GLE event of 1956 (green line). Even though we assume these high particle fluxes to be constant in our steady-state assumption, this result 


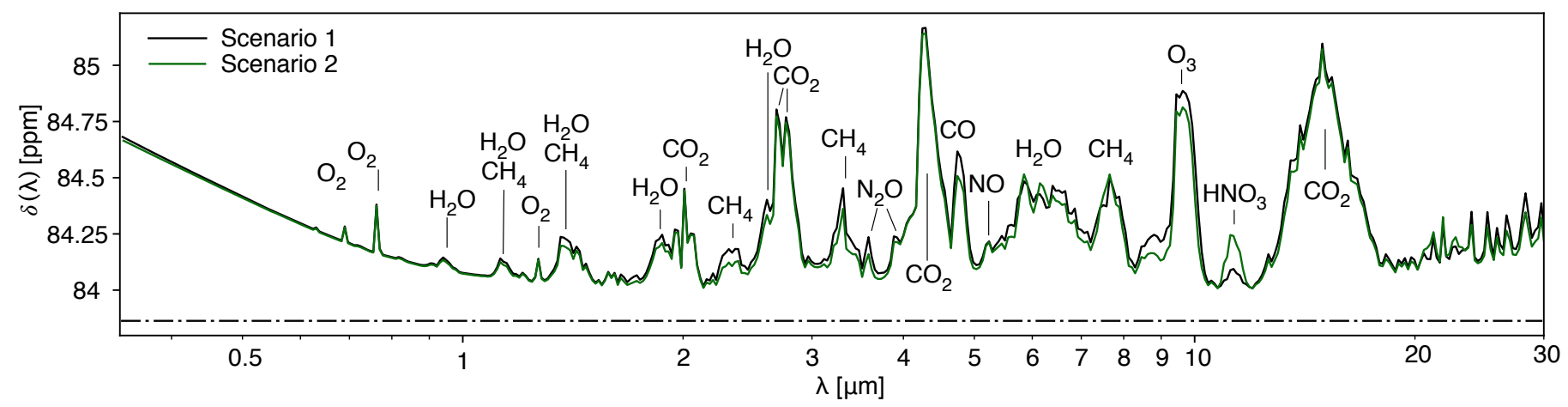

Fig. 12. Modeled transit depths in parts per million over wavelength $\lambda(R=100)$ for Earth around our G-type star. Shown are the results of scenario 1 (quiescent Sun, in black) and scenario 2 (GLE05, in green). The dashed-dotted black line at the bottom represents the transit depth for Earth without atmosphere (83.7 ppm).
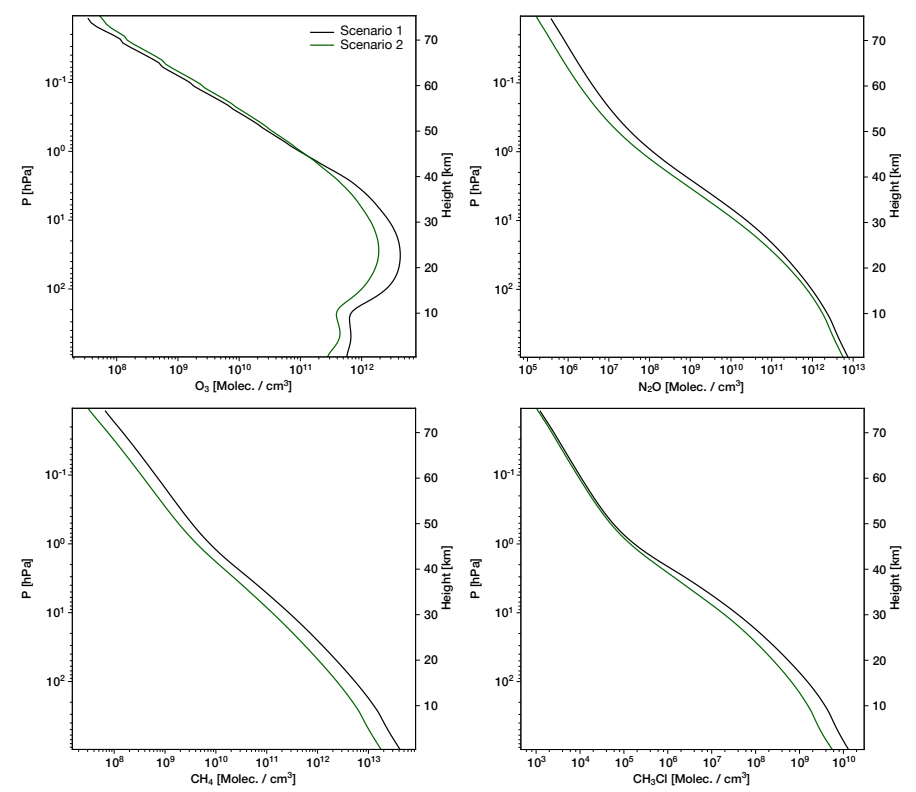

Fig. 13. Atmospheric profiles of the atmospheric biosignatures commonly discussed in exoplanetary literature: $\mathrm{O}_{3}$ (upper left panel), $\mathrm{N}_{2} \mathrm{O}$ (upper right panel), $\mathrm{CH}_{4}$ (lower left panel), and $\mathrm{CH}_{3} \mathrm{Cl}$ (lower right panel). Shown are the results of scenario 1 (quiescent Sun, black lines) and scenario 2 (GLE05, green lines).

compares well with the measured $\mathrm{O}_{3}$ depletion after such big events at higher latitudes for a few months (see, e.g., McConnell et al. 2017). The effect of GLE05 on other molecules that are not shown here is however, overall, only minor throughout our model atmosphere.

\section{Summary and conclusions}

Here we present the validation of our self-consistent model chain to determine the influence of CRs on (exo)planetary atmospheric biosignatures based on the example of Earth as an exoplanet in the HZ of a G-type star.

We focused on the modeling of the impact of the stellar radiation and particle environment on exoplanetary atmospheric climate and chemistry, as well as on potential atmospheric biosignatures for the modern Earth as an example of an Earthlike exoplanet in the habitable zone of a G-type star. Numerical models developed and/or updated at the three institutions were used in order to build a self-consistent simulation chain. The chain takes into account the GCR and SCR flux, the particle transport and interaction within the planetary magnetic field (using PLANETOCOSMICS), the atmosphere by producing secondary particle cascades, and thus atmospheric ionization and radiation (using AtRIS), as well as the CR-induced influence on the atmospheric chemistry (1D-TUB) and the ion-chemistry (ExoTIC).

Energetic particles are known to have a strong influence on atmospheric ion chemistry, and we showed that it is mandatory to iterate between the different models in order to assure convergence between the different codes. While for GCRs only one iteration between the different models was necessary, in the case of the strongest GLE event ever measured (GLE05), at least three iteration rounds were needed until the modeled ionization rates converged (see Fig. 5). We showed that the GCR-induced atmospheric ionization is roughly two orders of magnitude lower at the Pfotzer maximum (16-20 km, see Fig. 6) than the GLE-induced values. As a consequence, also the reduction in the production rates of, for example, $\mathrm{NO}, \mathrm{H}$, or $\mathrm{OH}$ due to the induced ion-chemistry processes is in the same order (see Fig. 7).

Based on these findings, we computed the influence of the induced photochemical processes on potential biosignatures. Using an algorithm for the determination of chemical pathways (PAP) the contributions of ozone loss cycles (see Fig. 8) and sources (see Fig. 9) were studied. The results are in agreement with the well-studied ozone-UV feedback (negative feedback, see, e.g., WMO 1994) which helps to maintain the ozone layer.

Moreover, the altitude-dependent absorbed and equivalent dose rates and the atmospheric UVA, UVB, and UVC profiles for both scenarios were computed. We showed that a strong GLE, such as that on February 23, 1956, not only has a substantial impact on for example the surface absorbed dose rate, which also increased by up to two orders of magnitude, but also impacts the UVB flux, which would result in a harmful flux of about $3.5 \mathrm{~W} \mathrm{~m}^{-2}$ at the planetary surface (for the influence of surface UVB upon biogeochemical feedbacks during Earth's evolution see, e.g., Gebauer et al. 2017).

Although the studied GLE event was rather modest compared to what has been observed for other Sun-like stars (see Notsu et al. 2019) and flaring M-dwarfs like Ad Leonis or Proxima Centauri (see, e.g., Herbst et al. 2019a), its impact on the atmospheric biosignatures is clearly visible (see Figs. 12 and 13). Thereby, $\mathrm{O}_{3}$ for example is decreased by up to $50 \%$ in the lower-mid stratosphere as well as the lower troposphere due to the influence of this strong SEP event. Taking into account that old Sun-like stars have been found to produce superflares with energies above $5 \times 10^{34}$ erg once every 2000 to $3000 \mathrm{yr}$ (see 
Notsu et al. 2019), it is fair to assume that stronger GLE events may occur on the Sun. Imprints of such events have already been found in the cosmogenic radionuclide records of ${ }^{10} \mathrm{Be},{ }^{14} \mathrm{C}$, and ${ }^{36} \mathrm{Cl}$ around AD774/5, AD992/3, and 660 BC (see, e.g., Miyake et al. 2012; Mekhaldi et al. 2015; O'Hare et al. 2019, respectively). Since such energetic flares could result in proton events about 200 times stronger than GLE05 (Herbst et al. 2019a), it is highly likely that the atmospheric $\mathrm{O}_{3}$ depletion would be much stronger. Even stronger flares with energies up to $10^{36} \mathrm{erg}$ $\left(10 \mathrm{~W} \mathrm{~m}^{-2}\right)$ have been detected on $\mathrm{K}$ - and M-stars (see, e.g., Candelaresi et al. 2014; Howard et al. 2018). Such flares could result in particle events that are more than five orders of magnitude stronger than GLE05 (Herbst et al. 2019a) which could drastically impact planetary habitability.

These results indicate that ozone is likely depleted in the $\mathrm{N}_{2}$ $\mathrm{O}_{2}$-dominated atmospheres of planets in the habitable zones of frequently flaring stars. Furthermore, due to the slow recovery of ozone after flare events, ozone might not be a good biomarker for planets orbiting stars with high flaring rates.

Initial modeling efforts to study the impact of such superflares on planetary habitability have recently been published by Yamashiki et al. (2019) and Airapetian et al. (2019). In addition, the newly selected ISSI Team "The Role Of Solar And Stellar Energetic Particles On (Exo)Planetary Habitability (ETERNAL)", led by Herbst and Grenfell, will bring together scientific expertise from the fields of solar, planetary, and exoplanetary science in order to determine the impact of the stellar particle and radiation field around main sequence stars on the atmospheric chemistry, climate, and habitability of exoplanets with $\mathrm{N}_{2}-\mathrm{O}_{2-}, \mathrm{CO}_{2-}, \mathrm{H}_{2-}$, and $\mathrm{H}_{2} \mathrm{O}$-dominated atmospheres in the $\mathrm{HZ}$ of their host stars.

Acknowledgements. We thank the German Research Foundation (DFG) for financial support via the project The Influence of Cosmic Rays on Exoplanetary Atmospheric Biosignatures (Project number 282759267). K.H., J.L.G., and B.H acknowledge the International Space Science Institute and the supported International Team 464: The Role Of Solar And Stellar Energetic Particles On (Exo)Planetary Habitability (ETERNAL, http: //www. issibern.ch/teams/ exoeternal/). K.H. and B.H. further acknowledge the supported International Team 441: High EneRgy sOlar partICle Events Analysis (HEROIC, http:// www.issibern.ch/teams/heroic/). F.S. is supported by the DFG project SCHR1125/3-1.

\section{References}

Airapetian, V. S., Barnes, R., Cohen, O., et al. 2019, Int. J. Astrobiol., 1

Banjac, S. 2018, Atmospheric Radiation Interaction Simulator (AtRIS), https://et-wiki.physik. uni-kiel.de/atris/atris

Banjac, S., Herbst, K., \& Heber, B. 2019a, J. Geophys. Res., 124, 50

Banjac, S., Berger, L., Burmeister, S., et al. 2019b, J. Space Weather Space Clim., 9, A14

Banjac, S., Heber, B., Herbst, K., Berger, L., \& Burmeister, S. 2019c, J. Geophys. Res., accepted, http://doi.org/10.1029/2019JA026622

Bates, D. R., \& Nicolet, M. 1950, J. Geophys. Res., 55, 301

Bazilevskaya, G. A., Usoskin, I. G., Flückiger, E. O., et al. 2008, Space Sci. Rev., 137, 149

Belov, A., Garcia, H., Kurt, V., \& Mavromichalaki, E. 2005, Cosmic Res., 43, 171

Belu, A. R., Selsis, F., Morales, J. C., et al. 2011, A\&A, 525, A83

Buccino, A. P., Lemarchand, G. A., \& Mauas, P. J. D. 2007, Icarus, 192, 582

Büsching, I., Kopp, A., Pohl, M., et al. 2005, ApJ, 619, 314

Caballero-Lopez, R. A., \& Moraal, H. 2004, J. Geophys. Res. Space Phys., 109, A01101

Candelaresi, S., Hillier, A., Maehara, H., Brandenburg, A., \& Shibata, K. 2014, ApJ, 792, 67

Chapman, S. 1930, Phys. Rev., 36, 1014

Christensen, U. R., \& Aubert, J. 2006, Geophys. J. Int., 166, 97

Cohen, O., Drake, J. J., Glocer, A., et al. 2014, ApJ, 790, 57

Cox, N. L. J., Arzoumanian, D., André, P., et al. 2016, A\&A, 590, A110

Crutzen, P. J. 1970, QJRMS, 96, 320
Crutzen, P. J., Isaksen, I. S. A., \& Reid, G. C. 1975, Science, 189, 457 Des Marais, D. J., Harwit, M. O., Jucks, K. W., et al. 2002, Astrobiology, 2, 153 Desorgher, L., Flückiger, E. O., \& Gurtner, M. 2006, in 36th COSPAR Scientific Assembly, 36

Dittmann, J. A., Irwin, J. M., Charbonneau, D., et al. 2017, Nature, 544, 333

do Nascimento, J. D., Jr., Vidotto, A. A., Petit, P., et al. 2016, ApJ, 820, L15

Dorman, L. I., Pustil'Nik, L. A., Sternlieb, A., et al. 2004, IEEE Trans. Plasma Sci., 32, 1478

Fichtner, H., Heber, B., Herbst, K., Kopp, A., \& Scherer, K. 2013, Solar Activity, the Heliosphere, Cosmic Rays and Their Impact on the Earth's Atmosphere, ed. F.-J. Lübken (Dordrecht: Springer Netherlands), 55

France, K., Froning, C. S., Linsky, J. L., et al. 2013, ApJ, 763, 149

France, K., Loyd, R. O. P., Youngblood, A., et al. 2016, ApJ, 820, 89

Froning, C. S., France, K., Loyd, R. O. P., et al. 2018, AAS Meeting Abstracts, 231, 111.05

Fujii, Y., Angerhausen, D., Deitrick, R., et al. 2018, Astrobiology, 18, 739

Funke, B., Baumgaertner, A., Calisto, M., et al. 2011, Atm. Chem. Phys., 11, 9089

Funke, B., Ball, W., Bender, S., et al. 2017, Atm. Chem. Phys., 17, 3573

García Muñoz, A., Koskinen, T. T., \& Lavvas, P. 2018, Upper Atmospheres and Ionospheres of Planets and Satellites, eds. H. J. Deeg, \& J. A. Belmonte (Cham: Springer International Publishing), 349

Gebauer, S., Grenfell, J., Stock, J., et al. 2017, Astrobiology, 17, 27

Gebauer, S., Grenfell, J., Lehmann, R., \& Rauer, H. 2018a, Astrobiology, 18, 856 Gebauer, S., Grenfell, J. L., Lehmann, R., \& Rauer, H. 2018b, ACS Earth Space Chem, 2, 1112

Gieseler, J., Heber, B., \& Herbst, K. 2017, J. Geophys. Res. Space Phys., 122, 10 , 964

Gillon, M., Triaud, A. H. M. J., Demory, B.-O., et al. 2017, Nature, 542, 456

Godolt, M., Grenfell, J. L., Hamann-Reinus, A., et al. 2015, Planet. Space Sci., 111,62

Gordon, M., Goldhagen, P., Rodbell, K., et al. 2004, IEEE Trans. Nucl. Sci., 51, 3427

Gordon, I., Rothman, L., Hill, C., et al. 2017, J. Quant. Spectr. Rad. Transf., 203,

Grenfell, J. L., Grießmeier, J.-M., Patzer, B., et al. 2007, Astrobiology, 7, 208

Grenfell, J., Gebauer, S., von Paris, P., et al. 2011, Icarus, 211, 81

Grenfell, J. L., Grießmeier, J.-M., von Paris, P., et al. 2012, Astrobiology, 12 1109

Grenfell, J. L., Gebauer, S., Godolt, M., et al. 2013a, Astrobiology, 13, 415

Grenfell, J. L., Stock, J. W., \& Patzer, A. B. C. 2013b, Planet. Space Sci., 84, 14

Grenfell, J., Gebauer, S., v. Paris, P., Godolt, M., \& Rauer, H. 2014, Planet. Space Sci., 98, 66

Grenfell, J. L., Gebauer, S., Godolt, M., et al. 2018, ApJ, 861, 38

Grießmeier, J.-M., Stadelmann, A., Grenfell, J., Lammer, H., \& Motschmann, U. 2009, Icarus, 199, 526

Guo, J., Banjac, S., Röstel, L., et al. 2019, J. Space Weather Space Clim., 9, A2 Haagen-Smit, A. J. 1952, Ind. Eng. Chem., 44, 1342

Haino, S., Sanuki, T., Abe, K., et al. 2004, Phys. Lett. B, 594, 35

Hedelt, P., von Paris, P., Godolt, M., et al. 2013, A\&A, 553, A9

Herbst, K., Kopp, A., \& Heber, B. 2013, Ann. Geophys., 31, 1637

Herbst, K., Muscheler, R., \& Heber, B. 2017, J. Geophys. Res. Space Phys., 122, 23

Herbst, K., Papaioannou, A., Banjac, S., \& Heber, B. 2019a, A\&A, 621, A67

Herbst, K., Banjac, S., \& Nordheim, T. A. 2019b, A\&A, 624, A124

Horneck, G. 2001, Adv. Space Res., 26, 1983

Hörst, S. M. 2017, J. Geophys. Res. Planets, 122, 432

Howard, W. S., Tilley, M. A., Corbett, H., et al. 2018, ApJ, 860, L30

Hu, Y., \& Yang, J. 2014, Proc. Natl. Acad. Sci., 111, 629

Hummel, J. R., \& Reck, R. A. 1979, J. Appl. Meteorol., 18, 239

ICRP 2007, ICRP Publication 103. Ann. ICRP, 37, 2

Izmodenov, V. V., \& Alexashov, D. B. 2015, ApJS, 220, 32

Jackman, C. H., Frederick, J. E., \& Stolarski, R. S. 1980, J. Geophys. Res., 85, 7495

Jackman, C. H., Douglass, A. R., Rood, R. B., McPeters, R. D., \& Meade, P. E. 1990, J. Geophys. Res., 95, 7417

Jackman, C. H., Fleming, E. L., \& Vitt, F. M. 2000, J. Geophys. Res., 105, 11659

Jackman, C. H., Deland, M. T., Labow, G. J., et al. 2005a, Adv. Space Res., 35, 445

Jackman, C. H., Deland, M. T., Labow, G. J., et al. 2005b, J Geophys. Res. Space Phys., 110, A09S27

Jackman, C. H., Marsh, D. R., Vitt, F. M., et al. 2009, J. Geophys. Res. Atm., 114, D11304

Jacquinet-Husson, N., Armante, R., Scott, N. A., et al. 2016, J. Mol. Spectr., 327, 31

Joshi, M. 2003, Astrobiology, 3, 415

Joshi, M. M., \& Haberle, R. M. 2012, Astrobiology, 12, 3

Joshi, M. M., Haberle, R. M., \& Reynolds, R. T. 1997, Icarus, 129, 450 
K. Herbst et al.: Influence of cosmic rays on exoplanetary atmospheric biosignatures

Jucks, K., Johnson, D., Chance, K., et al. 1996, J. Geophys. Res., 101, 28785 Kaltenegger, L., \& Traub, W. A. 2009, ApJ, 698, 519

Kasting, J. F., Pollack, J. B., \& Ackerman, T. P. 1984, Icarus, 57, 335

Katushkina, O. A., Alexashov, D. B., \& Gvaramadze, V. V. 2018, MNRAS, 473,

Keppler, F., Harper, D. B., Röckmann, T., Moore, R. M., \& Hamilton, J. T. G. 2005, Atm. Chem. Phys., 5, 2403

Khodachenko, M. L., Ribas, I., Lammer, H., et al. 2007, Astrobiology, 7, 167

Kissmann, R., Kleimann, J., Fichtner, H., \& Grauer, R. 2008, MNRAS, 391, 4

Kobayashi, K., Geppert, W. D., Carrasco, N., et al. 2017, Astrobiology, 17, 786

Kobulnicky, H. A., Chick, W. T., Schurhammer, D. P., et al. 2016, ApJS, 227, 18

Kopp, A., Schilp, S., \& Preusse, S. 2011, ApJ, 729, 116

Kopparapu, R. k., Wolf, E. T., Haqq-Misra, J., et al. 2016, ApJ, 819, 84

Kremer, J., Boezio, M., Ambriola, M. e., et al. 1999, Phys. Rev. Lett., 83, 4241

Lammer, H., Bredehöft, J. H., Coustenis, A., et al. 2009, A\&ARv, 17, 181

Lavvas, P., Yelle, R. V., Koskinen, T., et al. 2013, Proc. Natl. Acad. Sci., 110, 2729

Leconte, J., Forget, F., Charnay, B., et al. 2013, A\&A, 554, A69

Lehmann, R. 2004, J. Atm. Chem., 47, 45

Manabe, S., \& Wetherald, R. T. 1967, J. Atm. Sci., 24, 241

McConnell, J. R., Burke, A., Dunbar, N. W., et al. 2017, Proc. Natl. Acad. Sci., 114,10035

Mekhaldi, F., Muscheler, R., Adolphi, F., et al. 2015, Nat. Commun., 6, 8611

Mironova, I. A., Aplin, K. L., Arnold, F., et al. 2015, Space Sci. Rev., 194, 1

Miyake, F., Nagaya, K., Masuda, K., \& Nakamura, T. 2012, Nature, 486, 240

Möller, T. 2013, PhD Thesis, Christian-Albrechts-Universität zu Kie

Moraal, H. 2013, Space Sci. Rev., 176, 299

Neher, H. 1971, J. Geophys. Res., 76, 1637

Nieder, H., Winkler, H., Marsh, D. R., \& Sinnhuber, M. 2014, J. Geophys. Res. Space Phys., 119, 2137

Noack, L., Snellen, I., \& Rauer, H. 2017, Space Sci. Rev., 212, 877

Notsu, Y., Maehara, H., Honda, S., et al. 2019, ApJS, 876, 58

O'Hare, P., Mekhaldi, F., Adolphi, F., et al. 2019, Proc. Natl. Acad. Sci., 116, 5961

Parker, E. N. 1968, J. Geophys. Res., 73, 6842

Pavlov, A. A., Kasting, J. F., Brown, L. L., Rages, K. A., \& Freedman, R. 2000 J. Geophys. Res. Planets, 105, 11981

Porter, H. S., Jackman, C. H., \& Green, A. E. S. 1976, J. Chem. Phys., 65, 154

Preusse, S., Kopp, A., Büchner, J., \& Motschmann, U. 2005, A\&A, 434, 1191

Preusse, S., Kopp, A., Büchner, J., \& Motschmann, U. 2006, A\&A, 460, 317

Rauer, H., Gebauer, S., von Paris, P. V., et al. 2011, A\&A, 529, A8

Raukunen, O., Vainio, R., Taylka, A. J., et al. 2018, J. Space Weather Space Clim., 8, A04

Rimmer, P., \& Rugheimer, S. 2019, Icarus, 329, 124

Rohen, G., von Savigny, C., Sinnhuber, M., et al. 2005, J. Geophys. Res. Space Phys., 110, A09S39

Rugheimer, S., Kaltenegger, L., Segura, A., Linsky, J., \& Mohanty, S. 2015, ApJ 809,57

Scalo, J., Kaltenegger, L., Segura, A. G., et al. 2007, Astrobiology, 7, 85

Scherer, K., van der Schyff, A., Bomans, D., et al. 2015, A\&A, 576, A97

Scheucher, M., Grenfell, J., Wunderlich, F., et al. 2018, ApJ, 863, 6

Schreier, F., Gimeno García, S., Hedelt, P., et al. 2014, J. Quant. Spectr. Rad. Transf., 137, 29

Schreier, F., Milz, M., Buehler, S. A., \& von Clarmann, T. 2018a, J. Quant. Spectr. Rad. Transf., 211, 64
Schreier, F., Städt, S., Hedelt, P., \& Godolt, M. 2018b, Mol. Astrophys., 11, 1 Schwieterman, E. W., Kiang, N. Y., Parenteau, M. N., et al. 2018, Astrobiology, 18,663

Segura, A., Krelove, K., Kasting, J., et al. 2003, Astrobiology, 3, 689

Segura, A., Kasting, J. F., Meadows, V., et al. 2005, Astrobiology, 5, 706

Segura, A., Walkoicz, L. M., Meadows, V., Kasting, J., \& Hawley, S. 2010, Astrobiology, 10, 751

Shea, M. A., \& Smart, D. F. 2000, Space Sci. Rev., 93, 229

Shields, A. L., Meadows, V. S., Bitz, C. M., et al. 2013, Astrobiology, 13, 715

Shields, A. L., Ballard, S., \& Johnson, J. A. 2016, Phys. Rep., 663, 1

Simon Wedlund, C., Gronoff, G., Lilensten, J., Ménager, H., \& Barthélemy, M. 2011, Ann. Geophys., 29, 187

Sinnhuber, B.-M., Weber, M., Amankwah, A., \& Burrows, J. P. 2003, Geophys. Res. Lett., 30, 1580

Sinnhuber, M., Nieder, H., \& Wieters, N. 2012, Surv. Geophys., 33, 1281

Snellen, I. A. G., de Kok, R. J., le Poole, R., Brogi, M., \& Birkby, J. 2013, ApJ, 764,182

Solomon, S., \& Crutzen, P. J. 1981, J. Geophys. Res., 86, 1140

Solomon, S., Reid, G. C., Rusch, D. W., \& Thomas, R. J. 1983, Geophys. Res. Lett., 10, 257

Stock, J. W., Boxe, C. S., Lehmann, R., et al. 2012, Icarus, 219, 13

Stone, E. C., Cummings, A. C., McDonald, F. B., et al. 2013, Science, 341, 150

Swider, W., \& Keneshea, T. J. 1973, Planet. Space Sci., 21, 1969

Syakila, A., \& Kroeze, C. 2011, Greenhouse Gas Meas. Manag., 1, 17

Tabataba-Vakili, F., Grenfell, J. L., Grießmeier, J. M., \& Rauer, H. 2016, A\&A, 585, A96

Tilley, M. A., Segura, A., Meadows, V., Hawley, S., \& Davenport, J. 2019, Astrobiology, 19, 64

Toon, O. B., McKay, C. P., Ackerman, T. P., \& Santhanam, K. 1989, J. Geophys. Res. Atm., 94, 16287

Tsyganenko, N. A., \& Stern, D. P. 1996, J. Geophys. Res. Space Phys., 101, 27187

Usoskin, I. G., Kovaltsov, G. A., Mironova, I. A., Tylka, A. J., \& Dietrich, W. F. 2011, Atm. Chem. Phys., 11, 1979

van Marle, A. J., Decin, L., \& Meliani, Z. 2014, A\&A, 561, A152

Vasquez, M., Gottwald, M., Gimeno García, S., et al. 2013a, Adv. Space Res., 51, 835

Vasquez, M., Schreier, F., Gimeno García, S., et al. 2013b, A\&A, 549, A26

Verronen, P. T., \& Lehmann, R. 2013, Ann. Geophys., 31, 909

Vidotto, A. A., Jardine, M., \& Helling, C. 2011, MNRAS, 411, L46

Vidotto, A. A., Jardine, M., Morin, J., et al. 2013, A\&A, 557, A67

von Paris, P., Gratier, P., Bordé, P., \& Selsis, F. 2016, A\&A, 587, A149

Vuitton, V., Yelle, R., \& McEwan, M. 2007, Icarus, 191, 722

Wahlen, M. 1993, Ann. Rev. Earth Planet. Sci., 21, 407

Webber, W. R., \& McDonald, F. B. 2013, Geophys. Res. Lett., 40, 1665

Wiengarten, T., Kleimann, J., Fichtner, H., et al. 2013, J. Geophys. Res. Space Phys., 118, 29

Winkler, H., Kazeminejad, S., Sinnhuber, M., Kallenrode, M. B., \& Notholt, J. 2009, J. Geophys. Res. Atm., 114, D00I03

WMO 1994, GORMP- No. 37

Wood, B. E., Redfield, S., Linsky, J. L., Müller, H.-R., \& Zank, G. P. 2005, ApJS, 159,118

Yamashiki, Y. A., Maehara, H., Airapetian, V., et al. 2019, ApJ, 881, 114

Yang, J., Cowan, N. B., \& Abbot, D. S. 2013, ApJS, 771, L45

Youngblood, A., France, K., Loyd, R. O. P., et al. 2017, ApJ, 843, 31

Zeitlin, C., Miller, J., Guetersloh, S., et al. 2011, Phys. Rev. C, 83, 034909 